\title{
Berberine Regulates Treg/Th17 Balance to Treat Ulcerative Colitis Through Modulating the Gut Microbiota in the Colon
}

OPEN ACCESS

Edited by:

Frank Wagener

Radboud Institute for Molecular Life

Sciences, Netherlands

Reviewed by:

Salah Amasheh,

Freie Universität Berlin, Germany

Claudio Ferrante,

Università degli Studi "G. d'Annunzio"

Chieti-Pescara, Italy

${ }^{*}$ Correspondence:

Yuhong Bian

bianyuhong_2012@163.com

${ }^{\dagger}$ These authors have contributed equally to this work.

Specialty section:

This article was submitted to Inflammation Pharmacology,

a section of the journal

Frontiers in Pharmacology

Received: 04 March 2018

Accepted: 14 May 2018

Published: 31 May 2018

Citation:

Cui H, Cai Y, Wang L, Jia B, Li J,

Zhao S, Chu $X$, Lin J, Zhang $X$, Bian $Y$ and Zhuang $P$ (2018)

Berberine Regulates Treg/Th17

Balance to Treat Ulcerative Colitis

Through Modulating the Gut

Microbiota in the Colon.

Front. Pharmacol. 9:571.

doi: 10.3389/fphar.2018.00571

\author{
Huantian Cui ${ }^{1+}$, Yuzi Cai't, Li Wang ${ }^{2 t}$, Beitian Jia', Junchen Li', Shuwu Zhao', \\ Xiaoqian Chu ${ }^{1}$, Jin Lin ${ }^{1}$, Xiaoyu Zhang ${ }^{1}$, Yuhong Bian ${ }^{1 *}$ and Pengwei Zhuang ${ }^{1}$ \\ ${ }^{1}$ Tianjin University of Traditional Chinese Medicine, Tianjin, China, ${ }^{2}$ Tianjin Second People's Hospital, Tianjin, China
}

Berberine (BBR), an alkaloid isolated from Rhizoma Coptidis, Cortex Phellode, and Berberis, has been widely used in the treatment of ulcerative colitis (UC). However, the mechanism of BBR on UC is unknown. In this study, we investigated the activities of T regulatory cell (Treg) and T helper 17 cell (Th17) in a dextran sulfate sodium (DSS)induced UC mouse model after BBR administration. We also investigated the changes of gut microbiota composition using $16 \mathrm{~S}$ rRNA analysis. We also examined whether BBR could regulate the Treg/Th17 balance by modifying gut microbiota. The mechanism was further confirmed by depleting gut microbiota through a combination of antibiotic treatment and fecal transplantations. Results showed that BBR treatment could improve the Treg/Th17 balance in the DSS-induced UC model. BBR also reduced diversity of the gut microbiota and interfered with the relative abundance of Desulfovibrio, Eubacterium, and Bacteroides. Moreover, BBR treatment did not influence the Treg/Th17 balance after the depletion of gut microbiota. Our results also revealed that fecal transplantation from BBR-treated mice could relieve UC and regulate the Treg/Th17 balance. In conclusion, our study provides evidence that BBR prevents UC by modifying gut microbiota and regulating the balance of Treg/Th17.

Keywords: ulcerative colitis, berberine, gut microbiota, Treg/Th17 balance, inflammation

\section{INTRODUCTION}

Ulcerative colitis (UC) is a chronic and nonspecific inflammatory disease that has a prolonged disease duration and is difficult to cure. The morbidity of UC has increased over the past few years. Long term effects of UC could lead to cancer (Matsunaga et al., 2017).

Currently, the pathogenesis of UC remains unclear. Hereditary factors, the gut environment, immune response, cell apoptosis, and infection have been implicated in UC. During UC progression, the T helper cell 17 (Th17) population, which contributes to inflammation, is usually increased, while T regulatory cells (Treg), which inhibit Th17 activity, are decreased (Luo et al., 2017). UC patients exhibit gut microbiota dysfunction which results in increased permeability of the intestinal epithelial cell barrier, triggering inflammatory response of the intestine (Hoque et al., 2000). Furthermore, probiotics, which could regulate the immune function and promote intestinal mucosa repair, are decreased in UC patients (Shen et al., 2014). Therefore, modulating gut microbiota to promote intestinal mucosa repair is of clinical importance in UC studies (Cui et al., 2004; Zhao H.M. et al., 2013). 
Adrenocortical hormone, 5-amino salicylic acid, and immunosuppressive drugs are commonly used in the current treatment of UC. However, these drugs have serious side-effects including intolerance or allergy responses (Di Paolo et al., 2001). Some medicinal plants have been demonstrated to have protective effects on UC. Microwave-assisted aqueous harpagophytum extract and hydroalcoholic extract of chamomile could relief UC through modulating the inflammatory response in a rat colon inflammatory model (Menghini et al., 2016; Locatelli et al., 2017). Traditional Chinese medicine (TCM) is widely used in the treatment of UC. Accumulated studies have shown that TCM plays an important role in regulating the gut microbiota during UC treatment. Gancaoxiexin decoction has been shown to increase the numbers of Lactobacillus and Bifidobacterium, and decrease the numbers of Escherichia coli in the intestine of UC patients (Zhao Q. et al., 2013). Interleukin (IL)-10 is produced by Treg and could suppress inflammatory response in UC (Jonuleit and Schmitt, 2003). Whereas $I L-33$ is produced by colonic epithelial cells and could drive a Th2-like cytokine response in UC patients (Seidelin et al., 2010). Tumor Necrosis Factor (TNF)- $\alpha$ is a proinflammatory cytokine and its level increased in UC patients (Rijnierse et al., 2006). Yiqishengyu decoction demonstrated a significant effect on UC by improving the microenvironment imbalance and modulating the $I L-10$ and IL-33 levels (Yao et al., 2013). Portulaca oleracea polysaccharide has also been shown to increase the amount of Lactobacillus and Bifidobacterium, increase the levels of $I L-10$, and decrease the TNF- $\alpha$ levels in the intestine of UC modelmice (Feng et al., 2015).

Berberine (BBR), an alkaloid isolated from Rhizoma Coptidis, Cortex Phellode, and Berberis, exhibits many biological functions and has been used in the treatment of diarrhea, gastroenteritis, diabetes, hyperlipidemia, cardiovascular diseases, and UC (Chen et al., 2014). Interestingly, it has been demonstrated that BBR prevents obesity and insulin resistance by modulating the gut microbiota (Zhang et al., 2012). Moreover, studies have demonstrated that probiotics such as Bifidobacterium fragilis could improve the mucosal immune system in UC through interaction with intestinal regulatory $\mathrm{T}$ cells (Treg) to induce IL-10 production (Smith et al., 2013; Luo et al., 2017). Based on these lines of evidence, we hypothesized that BBR could modulate the gut microbiota and regulate the Treg/Th17 balance in UC. In this study, we first investigated whether BBR could influence the Treg/Th17 balance in UC model mice. We then investigated the changes of gut microbiota composition using $16 \mathrm{~S}$ rRNA analysis after BBR treatment. Gut microbiota were depleted using a combination of antibiotics. Fecal transplantations were used to further confirm that BBR regulates the Treg/Th17 balance to prevent UC by modulating the gut microbiota.

\section{MATERIALS AND METHODS}

\section{Reagents}

The standard of berberine $\left(\mathrm{C}_{20} \mathrm{H}_{18} \mathrm{NO}_{4}\right.$, molecular weight: $235.324 \mathrm{kD}$ ) was obtained from Solarbio Biotechnology Co.,
Ltd. (Beijing, China). Dextran sulfate sodium (DSS molecular weight: 36000-50000kD), ciprofloxacin, metronidazole, Phorbol 12-myristate (PMA), ionomycin, and Bravertin A were also obtained from Solarbio Biotechnology Co., Ltd. (Beijing, China). Total DNA extraction kit, total RNA extraction kit, first-stand cDNA reverse transcription kit, polymerase chain reaction kit, and primers were obtained from TianGen Biotechnology Co., Ltd. (Beijing, China). Mouse IL-10 and IL-17 ELISA kits were obtained from Multi Science Biotechnology Co., Ltd. (Hangzhou, China). APC anti-CD4, FITC anti-IL17A, PE anti-CD25, and Alexa Fluor 488 anti-Foxp3 antibodies for flow cytometry were purchased from BD bioscience Co., Ltd. (Franklin Lakes, NJ, United States). BCA, alanine aminotransferase (ALT), aspartate aminotransferase (AST), blood urea nitrogen (BUN), and creatinine (Cr) test kit were purchased from Nanjing Jiancheng Biological Engineering Institute (Nanjing, China).

\section{Animals}

Thirty- to forty-day-old female Balb/c mice (18-22 g), were purchased from Beijing Huafukang Animal Company. All animals were handled using experimental protocols outlined by National Institutes of Health regulations and approved by the Ethics Committee and Use Committee of the Tianjin University of Traditional Chinese Medicine. Throughout the acclimatization and study periods, all animals had access to food and water ad libitum and were maintained on a $12 \mathrm{~h}$ light/dark cycle $\left(21 \pm 2{ }^{\circ} \mathrm{C}\right.$ with a relative humidity of $45 \pm 10 \%)$.

\section{Induction of UC Using DSS}

Ulcerative colitis was induced by administrating mice with $4 \%$ $(\mathrm{w} / \mathrm{v})$ DSS in the drinking water for 7 days. Thereafter, mice were provided with regular water for 3 days (Wang et al., 2016).

\section{Depletion of the Gut Microbiota}

Mice were given a combination of ciprofloxacin $(0.2 \mathrm{~g} / \mathrm{l})$ and metronidazole $(1 \mathrm{~g} / \mathrm{l})$ for 3 weeks in their drinking water (Elinav et al., 2011). Fecal samples were collected, and the total DNA of the samples was assessed for gut microbiota depletion. Liver tissues were weighed to measure the liver index. In addition, liver, kidney, small intestine, and colon were stained with hematoxylin and eosin (H\&E) and serum levels of AST, ALT, Cr, and BUN were also measured to evaluate the effect of antibiotic treatment on liver and kidney function.

\section{Animal Grouping}

Mice were randomly divided into three groups ( $n=10$ per group): DSS-BBR-, DSS+BBR-, and DSS+BBR+. The DSS+BBRand $\mathrm{DSS}+\mathrm{BBR}+$ groups received $\mathrm{DSS}$ diluted in drinking water to induce UC. Mice in the DSS+BBR+ group were treated with BBR (40 mg/kg) whereas mice in the DSS-BBR-group and DSS+BBR - group were treated with $0.2 \mathrm{~mL}$ normal saline orally once per day.

For the gut microbiota depletion experiment, mice were randomly divided into three groups $(n=10$ per group): 
antibiotics-DSS-BBR-, antibiotics + DSS + BBR-, and antibiotics+DSS+BBR+. Mice in the antibiotics-DSS-BBRgroup remained untreated; mice in the antibiotics + DSS $+B B R-$ and antibiotics+DSS+BBR+ groups were treated with a combination of antibiotics to deplete the gut microbiota followed by administration of DSS diluted in drinking water to induce UC. Meanwhile, mice in the antibiotics+DSS+BBR+ group were treated with BBR $(40 \mathrm{mg} / \mathrm{kg}$ ) orally once per day during DSS treatment whereas mice in the antibiotics-DSS-BBR- and the antibiotics+DSS+BBR- groups were treated with $0.2 \mathrm{~mL}$ normal saline orally during DSS treatment.

The fecal transplantation experiment was performed based on an established protocol (Borody et al., 2013) (Figure 1). In brief, donor mice were randomly divided into four groups $(n=6$ per group) including DSS-BBR-, DSS-BBR+, DSS+BBR-, and DSS+BBR+. The DSS-BBR- group was fed normally; the DSS-BBR+ group received BBR treatment $(40 \mathrm{mg} / \mathrm{kg})$ orally once per day; and the DSS+BBR- and DSS+BBR+ groups received DSS diluted in drinking water to induce UC. The DSS+BBR+ group received $\mathrm{BBR}$ treatment $(40 \mathrm{mg} / \mathrm{kg})$ orally once per day whereas the DSS+BBR - group received $0.2 \mathrm{~mL}$ normal saline treatment. After 10 days, stools from each group were collected daily from donor mice for the subsequent 10 days under a laminar flow hood in sterile conditions. Stools from donor mice of each group were pooled and $100 \mathrm{mg}$ stools were re-suspended in $1 \mathrm{ml}$ of sterile normal saline. The solution was vigorously mixed for $10 \mathrm{~s}$ using a bench top vortex followed by centrifugation at $800 \mathrm{~g}$ for $3 \mathrm{~min}$. The supernatant was collected and used as transplant material as described below. Fresh transplant material was prepared on the same day of transplantation within $10 \mathrm{~min}$ before oral gavage to prevent changes in bacterial composition. Recipient mice were randomly divided into four groups ( $n=7$ per group). Each group of recipient mice received DSS treatment to induce $\mathrm{UC}$ and $100 \mu \mathrm{l}$ of fresh transplant material orally per day for 10 days.

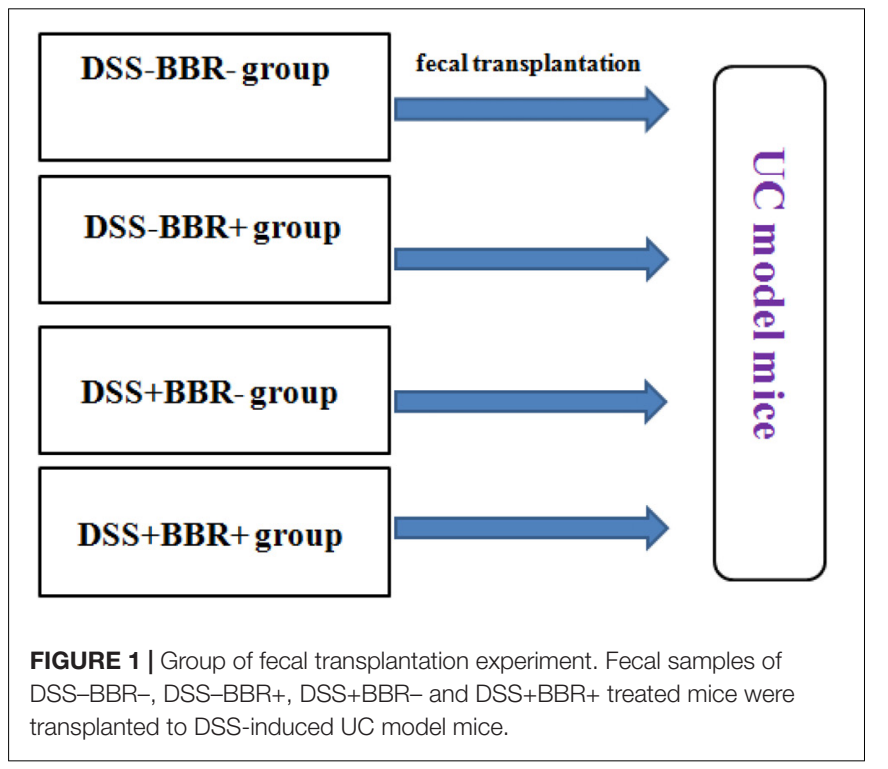

\section{Disease Activity Index (DAl)}

The severity of UC in each group was measured using DAI score. DAI is the combined score of body weight loss, stool consistency, and rectal bleeding, as in Table $\mathbf{1}$ and described previously (Kihara et al., 2003). The scores of DAI ranged from 0 (healthy) to 4 (severe colitis). The historical score is correlated with the pathologic findings in the DSS-induced model.

\section{Histology}

Mice liver, spleen, kidney, small intestine, and colon were removed and fixed in paraformaldehyde. The colon was then paraffin embedded and subsequently cut into $5 \mu \mathrm{M}$ sections. Sections were stained with hematoxylin and eosin (H\&E), the extent of inflammation was determined through a histological colitis score based on a previous study (Kihara et al., 2003). Scores ranged from 0 to 14 (total score), which represents the sum of scores from 0 to 4 for severity of extent, damage, inflammation, and regeneration (Table 2 ).

TABLE 1 | Disease activity index (DAl) (Kihara et al., 2003).

\begin{tabular}{llll}
\hline Score & Weight loss & Stool consistency & Rectal bleeding \\
\hline 0 & $<1 \%$ & Normal & Negative \\
1 & $5-10 \%$ & & \\
2 & $10-15 \%$ & Loose stool & Hemoccult + \\
3 & $15-20 \%$ & & \\
4 & $>20 \%$ & Diarrhea & Gross bleeding \\
\hline
\end{tabular}

Clinical criteria were used to evaluate the grade and extent of intestinal inflammation. Scores were tallied for each category and then divided by three to obtain the DAl.

TABLE 2 | Histological colitis score (Kihara et al., 2003).

\begin{tabular}{lll}
\hline Feature score & Score & Description \\
\hline Inflammation severity & 0 & None \\
& 1 & Mild \\
Inflammation extent & 3 & Moderate \\
& 0 & Severe \\
Crypt damage & 1 & None \\
& 2 & Mucosa \\
& 3 & Submucosa \\
& 0 & Transmural \\
& 1 & None \\
& 2 & Basal 1/3 damage \\
& 3 & Basal 2/3 damage \\
& 4 & Crypt lost; surface epithelium present \\
& 0 & Crypt and surface epithelium lost \\
& 1 & $0 \%$ \\
2 & 3 & $1-25 \%$ \\
& 4 & $26-50 \%$ \\
& & $51-75 \%$ \\
& & $76-100 \%$ \\
& &
\end{tabular}

The severity of inflammation was evaluated by histological colitis score. The score ranged from 0 to 14 (total score), which represents the sum of scores from 0 to 4 for severity and extent of inflammation, crypt damage, and percent of the colon involved. 


\section{Cytokine Quantification by Enzyme-Linked Immunoassay (ELISA)}

Colon tissue $(0.1 \mathrm{~g})$ was weighed and put into $900 \mu \mathrm{l}$ normal saline followed by ultrasonic trituration and centrifugation at $3000 \mathrm{rpm}$ for $15 \mathrm{~min}$ to obtain colon tissue homogenate. The levels of $I L-10$ and $I L-17$ in the colon tissue homogenate were measured by ELISA according to the manufacturer's instructions (Multi Science Biotechnology Co., China). Tissue homogenates were normalized to total protein content as detected by BCA assay (Nanjing Jiancheng Bioengineering Institute). The absorbance value was detected using a microplate reader.

\section{RNA Isolation and Real-Time Reverse Transcription Quantitative Polymerase Chain Reaction (RT-PCR)}

Total RNA was isolated from mice colon mucosa using the RNA extraction kit and first strand cDNA was synthesized from $1 \mu \mathrm{g}$ of total RNA according to the manufacturer's instructions. Quantitative RT-PCR (qRT-PCR) was used to detect the expression of $\beta$-actin, IL-10, IL-17, Fox3p, and ROR $\gamma t$ as previously described (Citri et al., 2011). All samples were run in triplicate and detected using a BIORAd iQ5 detection system. $\beta$-actin was used as a loading control. Quantification was undertaken using the $2^{-\Delta \Delta C_{\mathrm{T}}}$ method (Livak and Schmittgen, 2001). The sequences of all primers are listed in Table 3.

\section{Flow Cytometry}

Spleens were placed in $5 \mathrm{~mL}$ lymphocyte separation solution after mice were sacrificed. Single-cell suspensions were prepared by grinding over a nylon membrane $(70-\mu \mathrm{m}$ pore size), and lymphocytes were isolated after centrifuging at $800 \mathrm{~g}$ for $30 \mathrm{~min}$. The isolated cells were collected and treated with PMA

TABLE 3 | Primer sequences of target genes for mice.

\begin{tabular}{|c|c|c|}
\hline Genes & Primer sequence $\left(5^{\prime}-3^{\prime}\right)$ & $\begin{array}{l}\text { Amplicon size } \\
\text { (bp) }\end{array}$ \\
\hline \multirow[t]{2}{*}{$\beta$-actin } & $\begin{array}{l}\text { Forward: GCT GTC CCT GTA TGC } \\
\text { CTC T }\end{array}$ & 461 \\
\hline & $\begin{array}{l}\text { Reverse: GGT CTT TAC GGA TGT } \\
\text { CAA CG }\end{array}$ & \\
\hline \multirow[t]{2}{*}{ IL-10 } & $\begin{array}{l}\text { Forward: TAA TAA GCT CCA AGA } \\
\text { CCA AG }\end{array}$ & 262 \\
\hline & $\begin{array}{l}\text { Reverse: TAG AAT GGG AAC TGA } \\
\text { GGT ATC }\end{array}$ & \\
\hline \multirow[t]{2}{*}{ IL-17 } & $\begin{array}{l}\text { Forward: GTC AAT GCG GAG GGA } \\
\text { AAG }\end{array}$ & 349 \\
\hline & $\begin{array}{l}\text { Reverse: CAC GAA GCA GTT TGG } \\
\text { GAC }\end{array}$ & \\
\hline \multirow[t]{2}{*}{ Foxp3 } & $\begin{array}{l}\text { Forward: CAG GAG AAA GCG GAT } \\
\text { ACC AAA TG }\end{array}$ & 366 \\
\hline & $\begin{array}{l}\text { Reverse: ATC TGT GAG GAC TAC } \\
\text { CGA GCC }\end{array}$ & \\
\hline \multirow[t]{2}{*}{$\mathrm{ROR} \gamma \mathrm{T}$} & $\begin{array}{l}\text { Forward: ACC TCC ACT GCC AGC } \\
\text { TGT GTG CTG TC }\end{array}$ & 440 \\
\hline & $\begin{array}{l}\text { Reverse: TCA TाT CTG CAC TTC } \\
\text { TGC ATG TAG ACT GTC CC }\end{array}$ & \\
\hline
\end{tabular}

(30 $\mathrm{ng} / \mathrm{mL})$, ionomycin $(1 \mu \mathrm{g} / \mathrm{mL})$, and BFA $(10 \mathrm{ng} / \mathrm{mL})$ for $4 \mathrm{~h}$. Standard intracellular cytokine staining was used as previously described (Harrington et al., 2005). APC anti-mouse CD4 and FITC anti-mouse IL-17A were used for Th17 cell staining, whereas APC anti-mouse CD4, PE anti-mouse CD25, and Alexa Fluor 488 anti-mouse Foxp3 were selected for Treg staining. Samples and data were collected and analyzed with a BD-related device and software (FACSCalibur and Cell Quest-Pro).

\section{Isolation of Total DNA From Feces}

Fresh stool samples were collected and weighed. Total DNA in stool was isolated using the DNA extraction kit (TIANGEN, China). The DNA in stools was quantified as previously described (Li et al., 2017).

\section{Fecal 16S rRNA Analysis}

DNA was diluted to $1 \mathrm{ng} / \mu \mathrm{L}$ using sterile water. The extracted DNA from each sample was used as template to amplify the V4 region of 16S rRNA genes of distinct regions (16SV4) with specific primers (515F: 5'-GTGCCAGCMGCCGCGGTAA3' 806R: 5'-GGACTACHVGGGTWTCTAAT-3'). All PCR reactions were carried out using Phusion ${ }^{\circledR}$ High-Fidelity PCR Master Mix (New England Biolabs). PCR products were mixed with same volume of $1 \mathrm{X}$ loading buffer (containing SYBR green) and detected with electrophoresis on a $2 \%$ agarose gel. Further experiments were conducted using samples with bright bands between 280 and $320 \mathrm{bp}$. The TruSeq ${ }^{\circledR}$ DNA PCR-Free Sample Preparation Kit (Illumina, United States) was used to generate sequencing libraries. The library quality was assessed on the Qubit@2.0 Fluorometer (Thermo Scientific) and Agilent Bioanalyzer 2100 system. Finally, the library was sequenced on a Thermo Fisher Scientific Ion S5 XL platform and $600 \mathrm{bp}$ single-end reads were generated.

\section{Statistical Analysis}

All data were analyzed using one way analysis of variance (ANOVA) followed by Newman-Keuls comparison multiple test with mean \pm standard deviation (mean $\pm \mathrm{SD}$ ) for the independent experiments. Statistical differences between the experimental groups and control were examined and statistical significance was determined at a $p$-value $<0.05$, using SPSS version 20.0. Curve-fitting was carried out using GraphPad Prism5.

\section{RESULTS}

\section{BBR Improved Treg/Th17 Balance in DSS-Induced UC Model Mice}

Ulcerative colitis developed in all mice after DSS administration, evidenced by continuous body weight loss, shortened colon length, and high DAI score (Figures 2A-C, respectively) compared with animals without DSS treatment $(P<0.01$, $P<0.05, P<0.01$, respectively). Remarkably, BBR treatment reduced body weight loss and decreased loss of colon length caused by DSS administration, shown by the fact that mice treated 
A

Body weight change(\%)

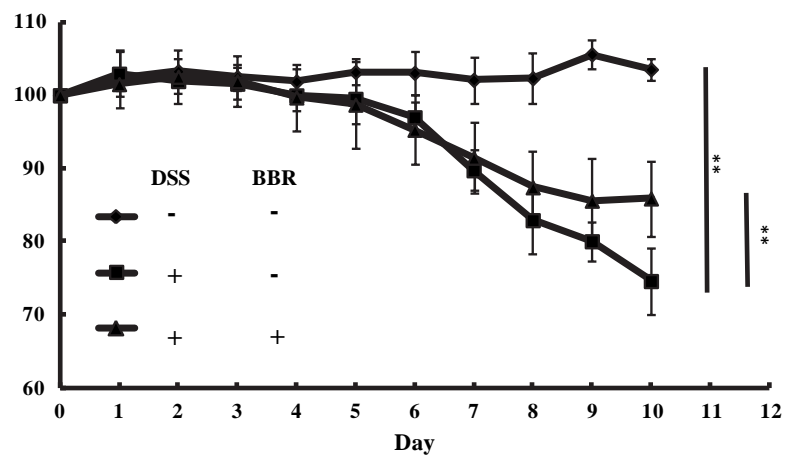

B

Colon Length(cm)

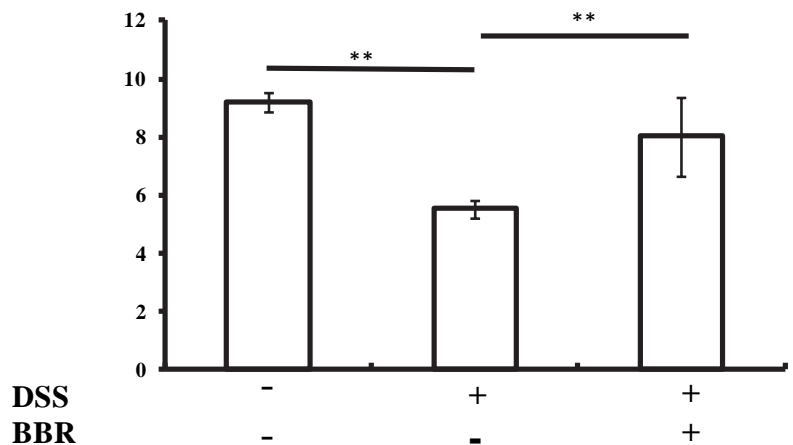

E Histological Colitis Score(0-14)

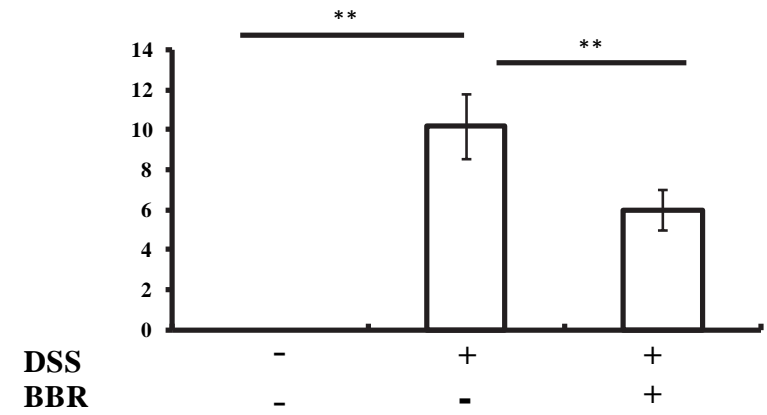

F

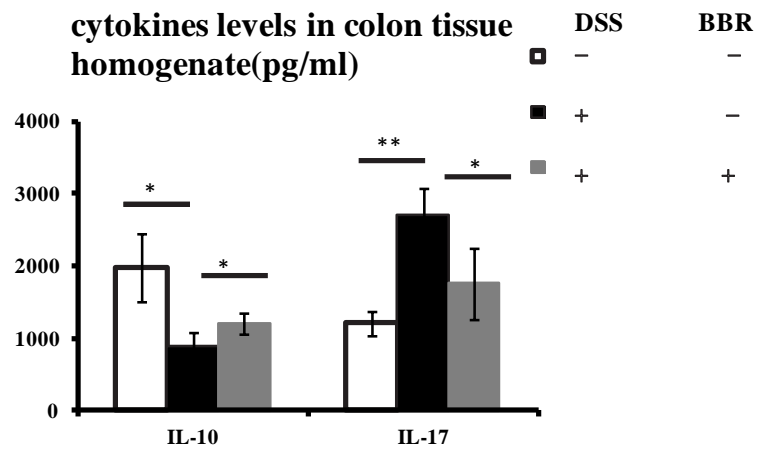

C

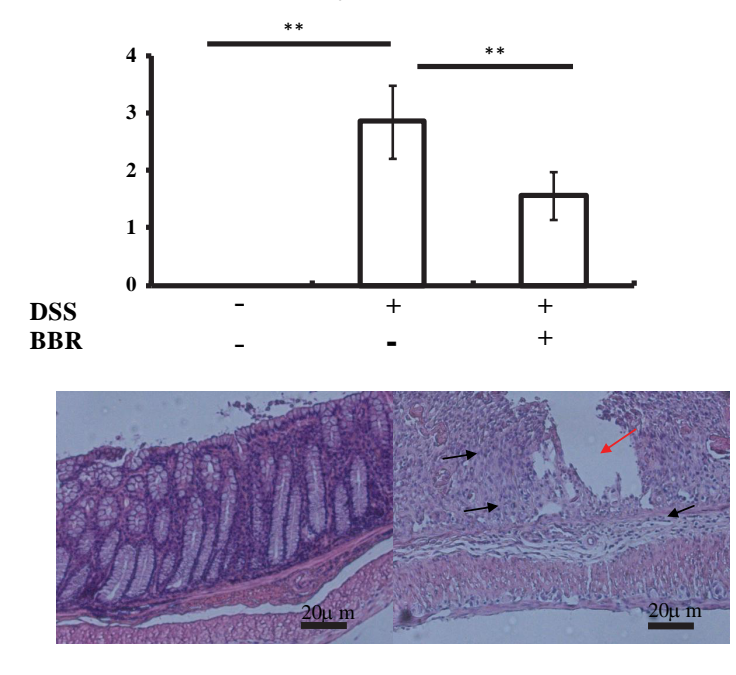

DSS - BBR -

DSS+ BBR -

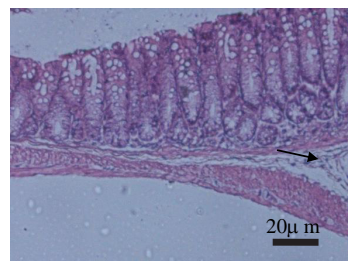

DSS + $\mathrm{BBR}+$

G Relative mRNA expression (fold to DSS+BBR- group)

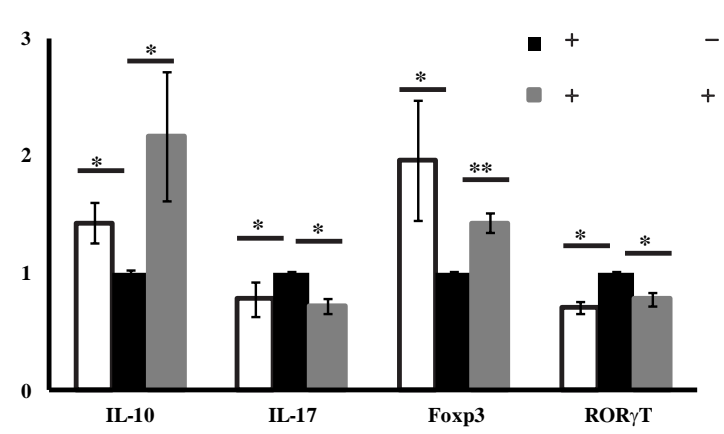


H a
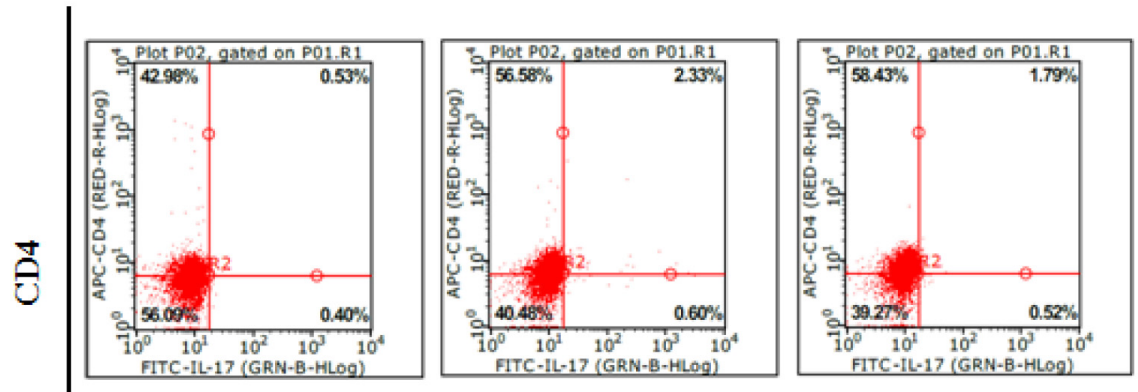

\section{IL-17A}

DSS

BBR

b
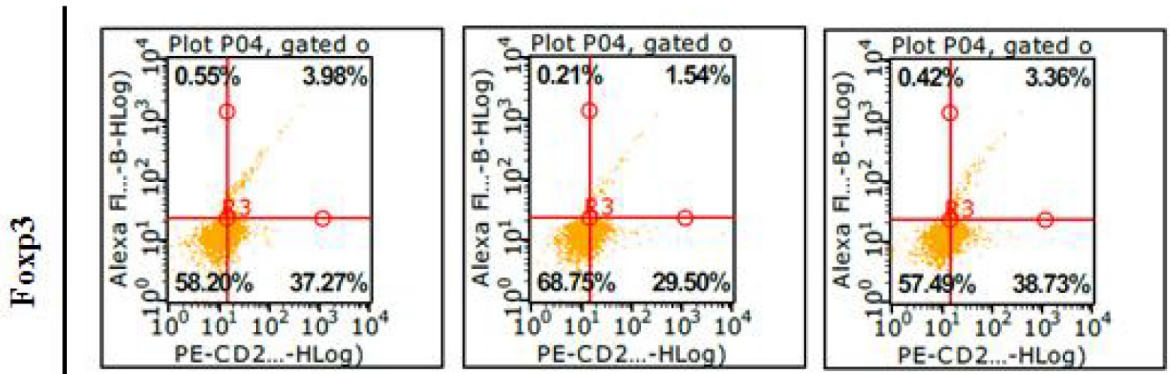

\section{CD25}

DSS

BBR

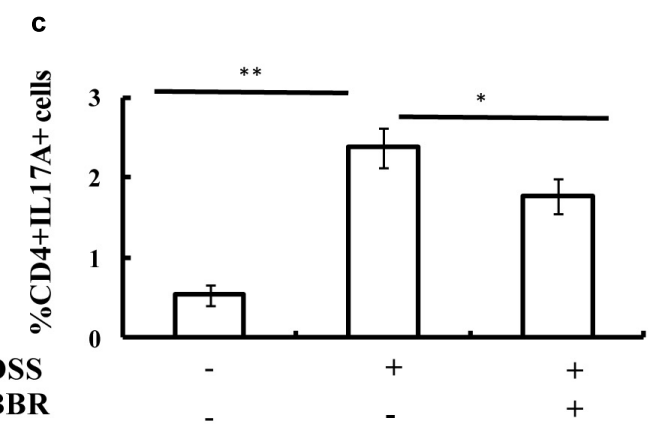

$+$
$+$

$+$

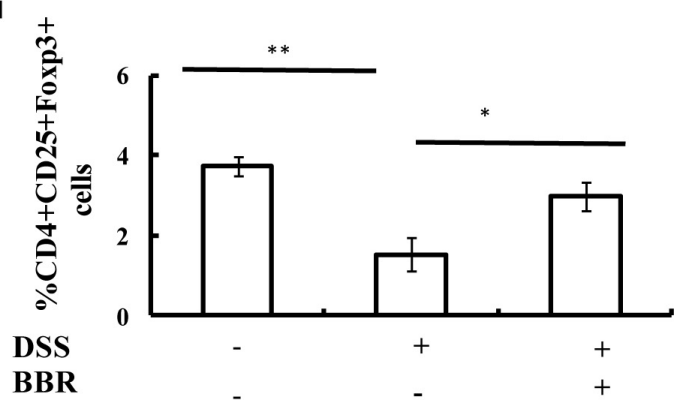

FIGURE 2 | Berberine (BBR) improved Treg/Thl7 balance in DSS-induced UC model mice. (A,B) Body weight loss and shorten of colon length were reduced after BBR treatment in DSS-induced UC model mice; (C) Mice treated with BBR had a lower DAl score compared with DSS treated mice; (D,E) H\&E staining (Arrows in red indicate mucosal necrosis, arrows in black indicate inflammatory cell infiltration; 20x) and histological colitis score showed that BBR reduced mucosal necrosis and inflammatory cell infiltration in colon; (F) BBR treatment increased $/ L-10$ and decreased $/ L$ - 17 levels of colon tissue homogenate in DSS-induced UC model mice; (G) After BBR treatment, the relative mRNA expression of $I L-10$ and Foxp3 in colonic mucosa was upregulated whereas the relative mRNA expression of $I L-17$ and $R O R \gamma T$ in colonic mucosa was downregulated as compared with DSS+BBR-group (Fold to DSS+BBR-group); (H) Flow cytometric analysis showed that BBR treatment decreased the numbers of CD4+IL17A+ (a: plot; c: graph) cells and increased the numbers of $\mathrm{CD}^{+}{ }^{+} \mathrm{CD}^{+} 5^{+}$Foxp3 ${ }^{+}(\mathbf{b}$ : plot; $\mathbf{d}$ : graph) in spleen compared with DSS+BBR- group. DSS-BBR-; DSS+BBR-; DSS+BBR+ ( $n=10$ per group, for A-G). DSS-BBR-; DSS+BBR-; DSS+BBR+ $(n=6$ per group, H). Data are presented as mean $\pm \mathrm{SD} .{ }^{*} p<0.05,{ }^{* *} p<0.01$ 
with BBR had a lower DAI score $(P<0.01)$. H\&E staining also showed that BBR relieved mucosal necrosis and infiltration of inflammatory cells triggered by DSS treatment, which resulted in lower histological colitis score compared with the model group $(P<0.05$, Figures $2 \mathrm{D}, \mathbf{E})$. Treg and Th17 activities were measured in each group. IL-10 levels in colonic tissue homogenate were decreased while $I L-17$ levels were increased in the DSS + BBR- group compared to the DSS-BBRgroup $(P<0.05, P<0.01$, respectively Figure $2 \mathrm{~F})$. BBR treatment increased $I L-10$ levels in colonic tissue homogenate and decreased colonic $I L-17$ levels compared to mice treated with DSS alone $(P<0.05$, Figure $2 \mathbf{F})$. In addition, $I L-10$ and Foxp3 gene expression were lower, while $I L-17$ and $R O R \gamma T$ gene expression were higher in colonic mucosa of DSS-treated mice compared with the DSS-BBR- group $(P<0.05, P<0.01$, $P<0.05, P<0.05$ respectively, Figure $2 \mathrm{G}$ ). The ratio of $\mathrm{CD}^{+} \mathrm{IL}_{17 \mathrm{~A}^{+}}$(Th17) cells in spleen lymphocytes were increased and the proportion of $\mathrm{CD} 4^{+} \mathrm{CD} 25^{+} \mathrm{Foxp}^{+}$(Treg) cells in spleen lymphocytes were decreased in the DSS+BBR- group compared with DSS - BBR - group $(P<0.01$, respectively, Figure $2 \mathrm{H})$. BBR treatment decreased the number of $\mathrm{CD} 4^{+} \mathrm{IL} 17 \mathrm{~A}^{+}$(Th17) cells and increased the number of $\mathrm{CD} 4^{+} \mathrm{CD} 25^{+} \mathrm{Foxp}^{+}$(Treg) cells in spleen lymphocytes $(P<0.05$, Figure $2 \mathbf{H})$.

\section{BBR Changed the Structure of Gut Microbiota in UC Model Mice}

A bar-coded pyrosequencing run was used to study the changes in gut microbiota from UC model mice after BBR treatment. In total, 1,454,065 useable reads and 1,577 OTUs were obtained from 18 samples. The Shannon indexes were lower in the DSS+BBR - and DSS+BBR+ groups compared with the DSS-BBR- group (Figure 3A). Moreover, a Venn diagram of the three groups revealed that 675 OTUs overlapped among the groups; 777 OTUs were present in the DSS-BBR- and DSS+BBR - groups; 710 in the DSS - BBR- and DSS+BBR+ groups; and 807 in the DSS+BBR- and DSS+BBR+ groups (Figure 3B). PCoA (principal co-ordinates analysis) showed that gut microbiota in the DSS+BBR-group were separated from the DSS-BBR- group, whereas the distance between the DSS+BBR+ and DSS-BBR- groups were closer than that between the DSS+BBR- and DSS-BBR- groups (Figure 3C). The system clustering tree also indicated a significant difference in each group and the distance from the DSS + BBR+ group to the DSS-BBR- group was small (Figure 3D).

We further investigated the gut microbiota species and their relative abundance. At the phylum level, 9 phyla could be found in all samples and the most abundant phyla in all samples were Bacteroidetes, Firmicutes, and Proteobacteria (Figure 3E). Conversely, Planctomycetes were detected in the DSS+BBRand DSS+BBR+ groups but not in the DSS-BBR- group (Figure 3E). Moreover, LefSe (LDA Effect Size) analysis was used to identify biomarkers and dominant microbiota in each group. The resulting cladogram revealed that Firmicutes were the major microbiota in the DSS-BBR- group. Proteobacteria, Streptococcaceae, Enterococcaceae, and Erysipelotrichale were predominant intestinal flora in the DSS+BBR- group and Bacteroidetes were predominant in the DSS+BBR+ group (Figure 3F). Furthermore, the relative abundance of Desulfovibrio and Lachnospiraceae_NK4A136_group were significantly higher whereas relative abundance of Eubacterium was remarkably lower in the DSS+BBR- group compared with the DSS-BBR- group (Figure 3G). The relative abundance of Desulfovibrio was decreased whereas the relative abundance of Eubacterium was increased in the DSS+BBR+ group compared with the DSS+BBR- group (Figure 3G). Interestingly, the relative abundance of Bacteroides in the DSS+BBR+ group was significantly increased compared with the DSS-BBRgroup and there were no significant differences in Bacteroides between the DSS+BBR- and DSS-BBR- groups (Figure 3G). In summary, our results showed that BBR could modulate gut microbiota composition in UC model mice.

\section{Modulation of Treg/Th17 Balance by BBR Following Depletion of Gut Microbiota}

A combination of antibiotics was used to deplete the gut microbiota followed by BBR treatment to study the effects on the Treg/Th17 balance (Elinav et al., 2011). Following the 3-week-antibiotic treatment, total DNA of gut microbiota in antibiotics-treated mice fecal samples were significantly lower than in mice that were not treated with antibiotics $(P<0.01$, Figure 4A). Moreover, H\&E staining showed that there were no morphological differences in liver, kidney, small intestine, or colon after antibiotics treatment compared to untreated mice (Figure 4B). There were no significant differences in liver index, colon length, or in serum levels of ALT, AST, Cr, and BUN after antibiotics treatment as compared to the untreated group, which indicated that antibiotics treatment depleted the gut microbiota. However, the liver and kidney function remained unaffected (Figure 4C).

In addition, following DSS treatment, antibiotics+DSS+BBR- treated mice showed significant body weight loss, shortened colon length and a high DAI score (Figures 5A-C, respectively) compared with mice with neither DSS nor antibiotics treatment $(P<0.01)$, whereas there were no significant differences between body weight loss, colon length, and DAI score in antibiotics+DSS+BBR+ treated mice compared with antibiotics+DSS+BBR- treated mice (Figures 5A-C). H\&E staining also demonstrated that BBR did not relieve mucosal necrosis or infiltration of inflammatory cells triggered by DSS treatment. In addition, there was no significant difference in histological colitis score between antibiotics+DSS+BBR+ treated mice compared with antibiotics+DSS+BBR- treated mice (Figures 5D,E).

Consistent with the earlier observations, $I L-10$ levels in colon tissue homogenate were decreased whilst $I L-17$ levels in colon tissue homogenate were increased in antibiotics+DSS+BBRtreated mice compared with antibiotics-DSS-BBR- treated mice $(P<0.05$, Figure 5F). Even though $I L-10$ levels in colon tissue homogenate were even lower in antibiotics+DSS+BBR+ treated mice compared with antibiotics+DSS+BBR- treated mice $(P<0.05$, Figure 5F), there was no significant difference between $I L-17$ levels in colon tissue homogenate 
A

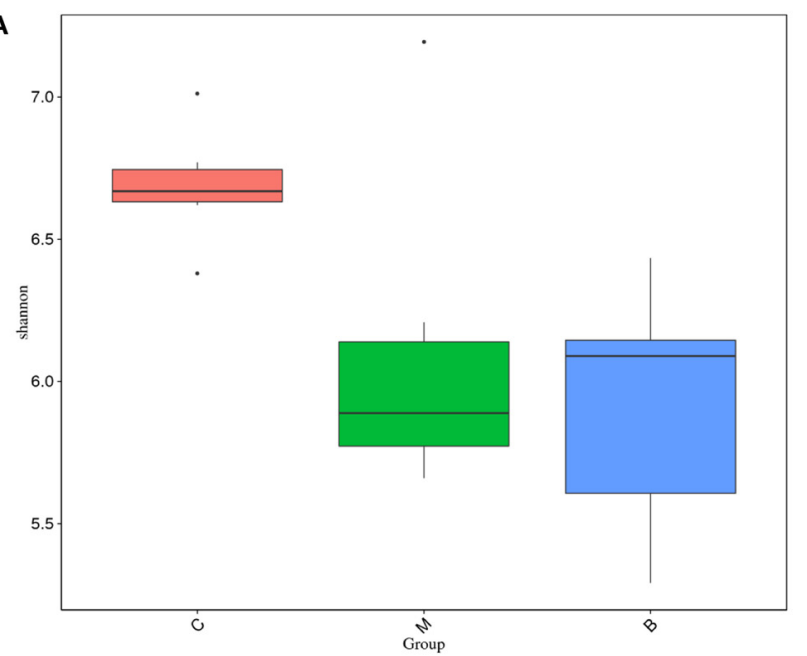

C

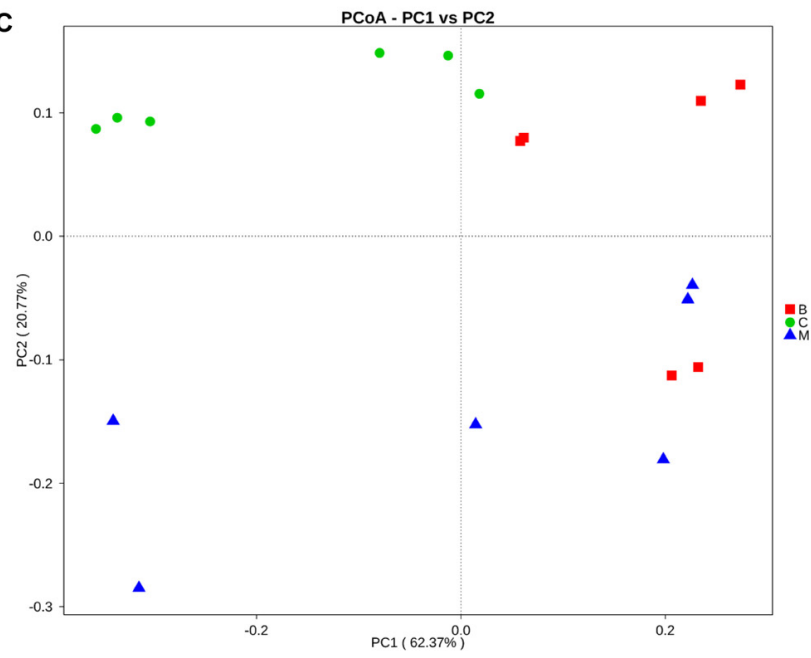

E

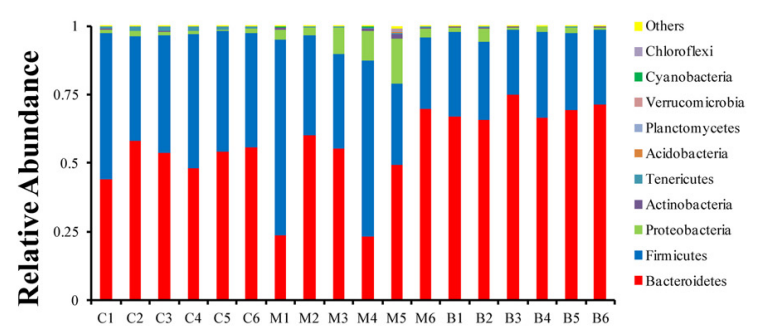

B

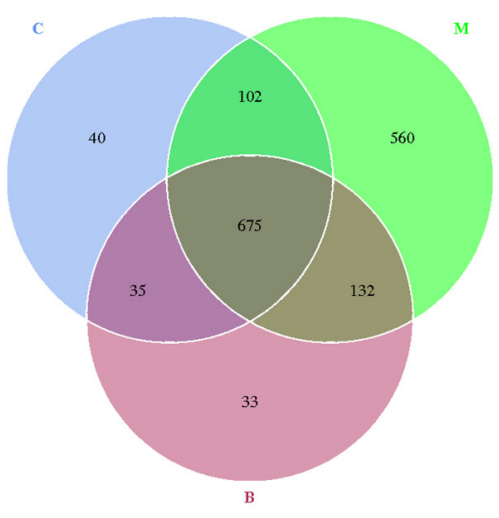

D

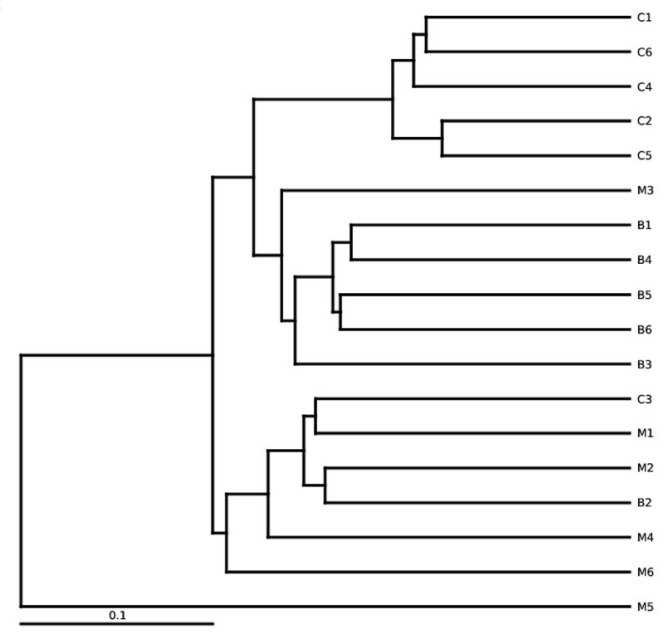

$\mathbf{F}$

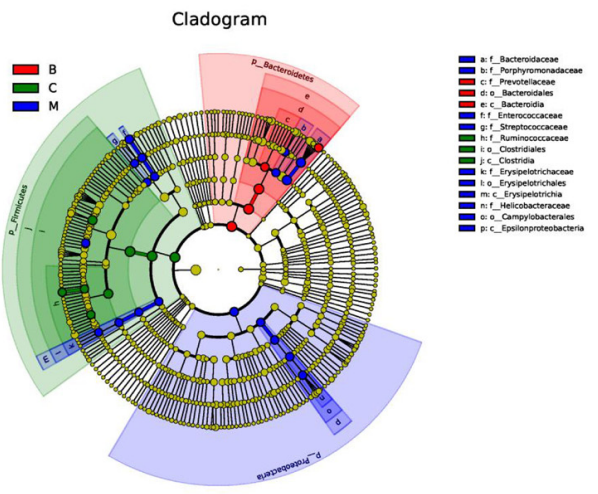

FIGURE 3 | Continued

in antibiotics $+\mathrm{DSS}+\mathrm{BBR}+$ treated mice compared with antibiotics + DSS + BBR - treated mice (Figure 5F). Colonic mucosa $I L-10$ and Foxp3 expression were lower, and $I L-17$ and $R O R \gamma T$ expression were higher in antibiotics+DSS+BBR- treated mice compared with mice with neither antibiotics nor DSS administration $(P<0.05$, Figure 5G), whereas there were no obvious differences in colonic mucosa expression of $I L-10$, Foxp3, IL-17, or ROR $\gamma T$ between antibiotics + DSS+BBR- 


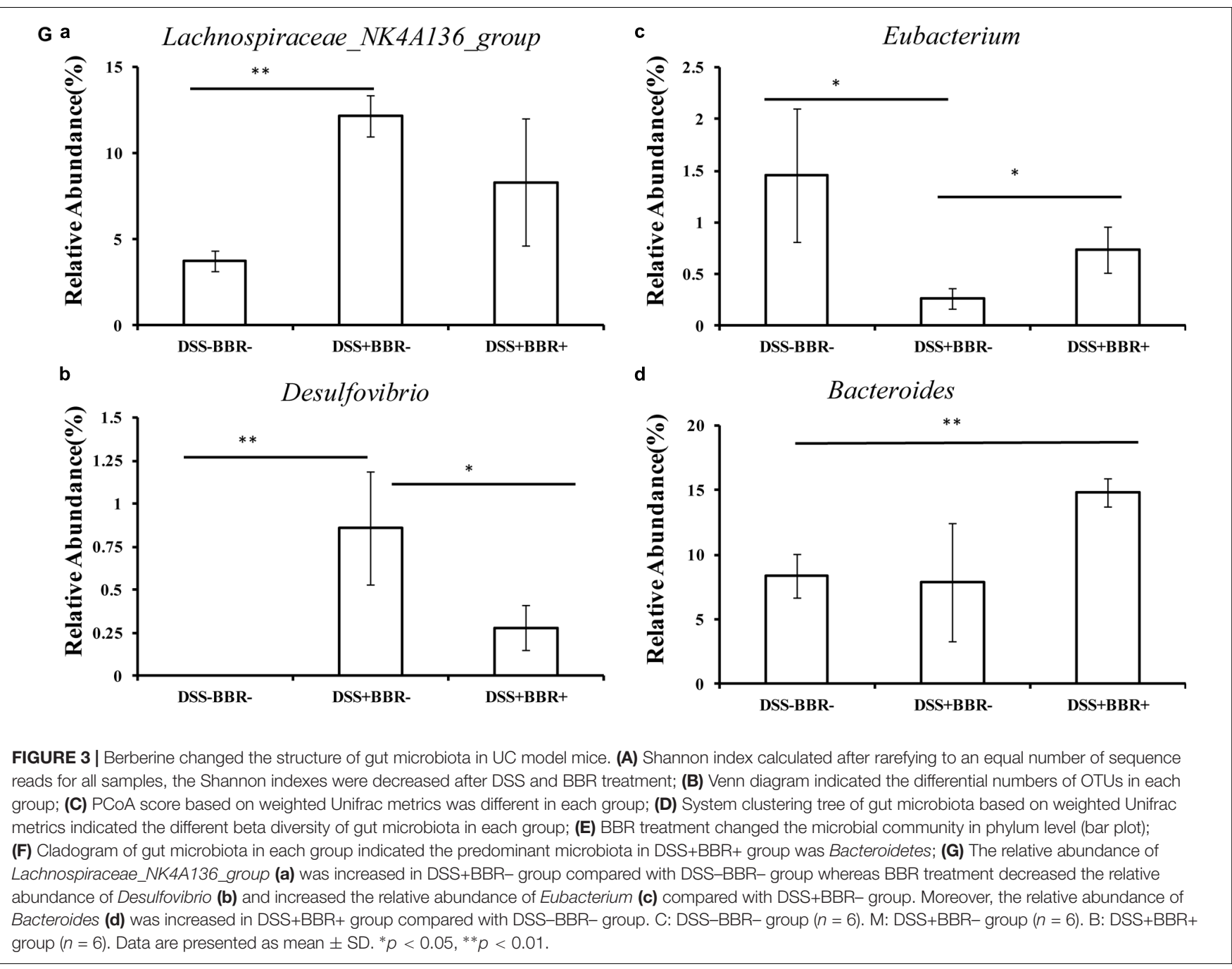

treated mice compared with antibiotics $+\mathrm{DSS}+\mathrm{BBR}+$ treated mice (Figure 5G). The number of $\mathrm{CD} 4^{+} \mathrm{IL}_{17 A^{+}}(\mathrm{Th} 17)$ cells in spleen lymphocytes were increased and the proportion of $\mathrm{CD} 4^{+} \mathrm{CD} 25^{+} \mathrm{Foxp}^{+}$(Treg) cells in spleen lymphocytes were decreased in antibiotics $+\mathrm{DSS}+\mathrm{BBR}-$ treated mice compared with mice in the antibiotics-DSS-BBR- group $(P<0.01$, Figure $5 \mathbf{H})$. There were no significant differences in the $\mathrm{CD}^{+}{ }^{+} \mathrm{IL}_{17 A^{+}}(\mathrm{Th} 17)$ and $\mathrm{CD}^{+} \mathrm{CD} 25+$ Foxp $^{+}$(Treg) cell ratio in spleen lymphocytes in antibiotics $+\mathrm{DSS}+\mathrm{BBR}-$ treated mice compared with antibiotics $+\mathrm{DSS}+\mathrm{BBR}+$ treated mice (Figure 5H).

\section{BBR Fecal Transplantation Reduced UC by Improving Treg/Th17 Balance}

Accumulated clinical studies have shown that fecal microbiota transplantation (FMT) could treat UC by modifying the gut microbiota (Borody et al., 2003; Anderson et al., 2012; Kunde et al., 2013; Moayyedi et al., 2015). To further confirm that BBR could improve the Treg/Th17 balance in the UC mice model by regulating the gut microbiota, gut microbiota of $\mathrm{DSS}-\mathrm{BBR}-, \mathrm{DSS}-\mathrm{BBR}+, \mathrm{DSS}+\mathrm{BBR}-$, and $\mathrm{DSS}+\mathrm{BBR}+$ treated mice were transferred into DSS-induced UC mice. UC mice that received fecal transplants from DSS-BBR-, $\mathrm{DSS}-\mathrm{BBR}+$ and $\mathrm{DSS}+\mathrm{BBR}+$ treated mice showed reduced body weight loss and DAI score compared with UC mice that received fecal matter from $\mathrm{DSS}+\mathrm{BBR}-$ mice (Figures $\mathbf{6 A}, \mathrm{C}$ ). In addition, UC mice receiving $\mathrm{DSS}-\mathrm{BBR}-$ and $\mathrm{DSS}-\mathrm{BBR}+$ fecal transplantations had a significantly increased colon length as compared with $\mathrm{UC}$ mice receiving $\mathrm{DSS}+\mathrm{BBR}$ - treated mice fecal transplantation (Figure 6B). H\&E staining showed that mucosal necrosis and infiltration of inflammatory cells were reduced in UC mice that received fecal matter from DSS-BBR-, $\mathrm{DSS}-\mathrm{BBR}+$, and $\mathrm{DSS}+\mathrm{BBR}+$ treated mice as compared with UC mice that received fecal matter from DSS+BBRtreated mice. This was evidenced by lower histological colitis scores compared with UC mice that received fecal matter from DSS+BBR - treated mice $(P<0.05$, Figures 6D,E). In addition, $I L-10$ levels in colonic tissue homogenate were increased in UC mice that received fecal transplants from DSS $-\mathrm{BBR}-$ and DSS-BBR+ treated mice compared to those that received fecal transplants from DSS+BBR- treated mice 


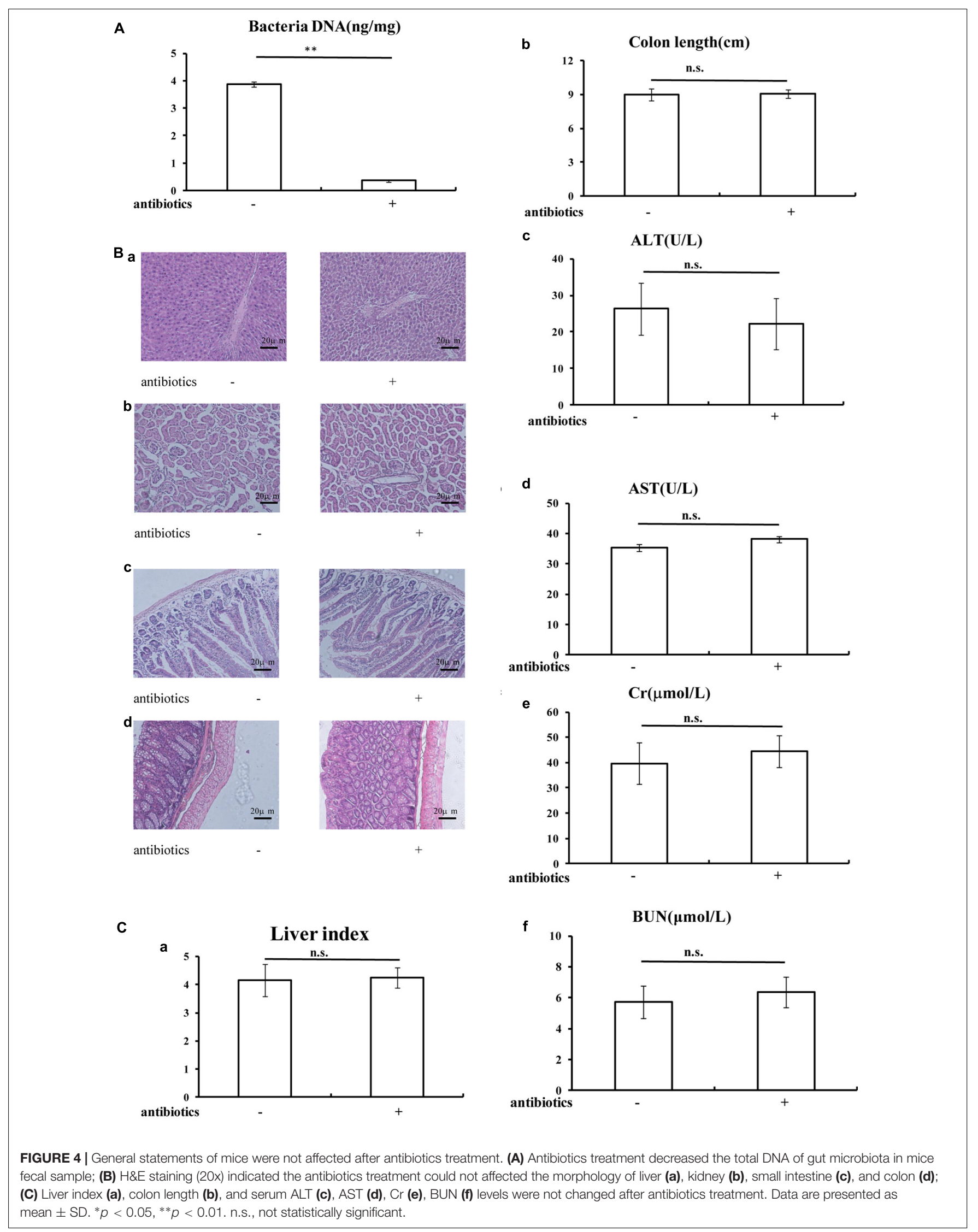




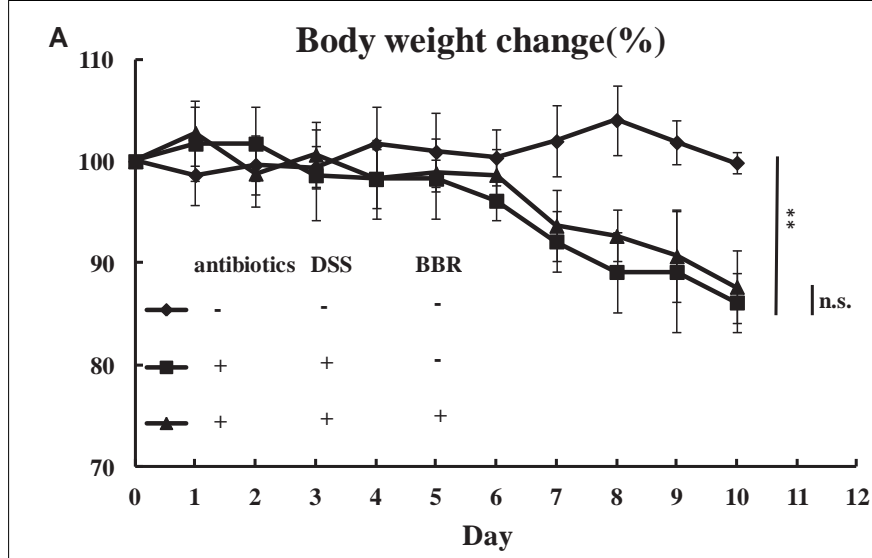

C

B

Colon length $(\mathrm{cm})$

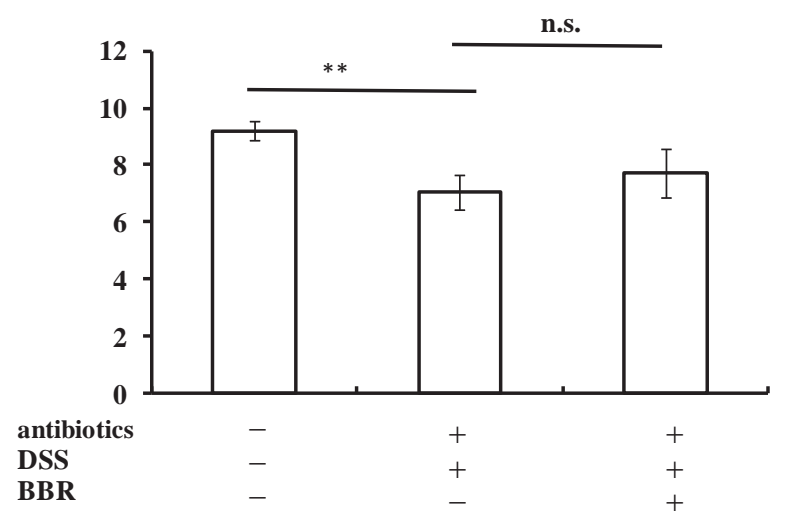

D
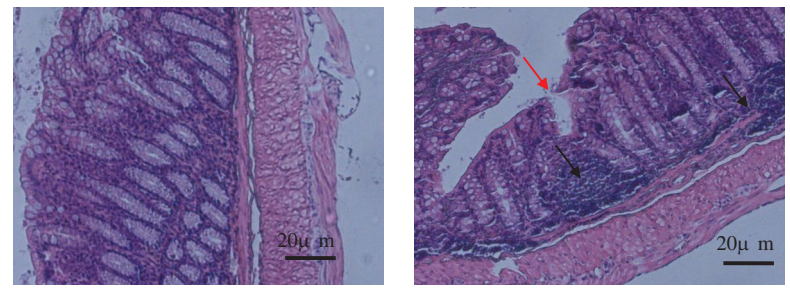

antibiotics

DSS

BBR
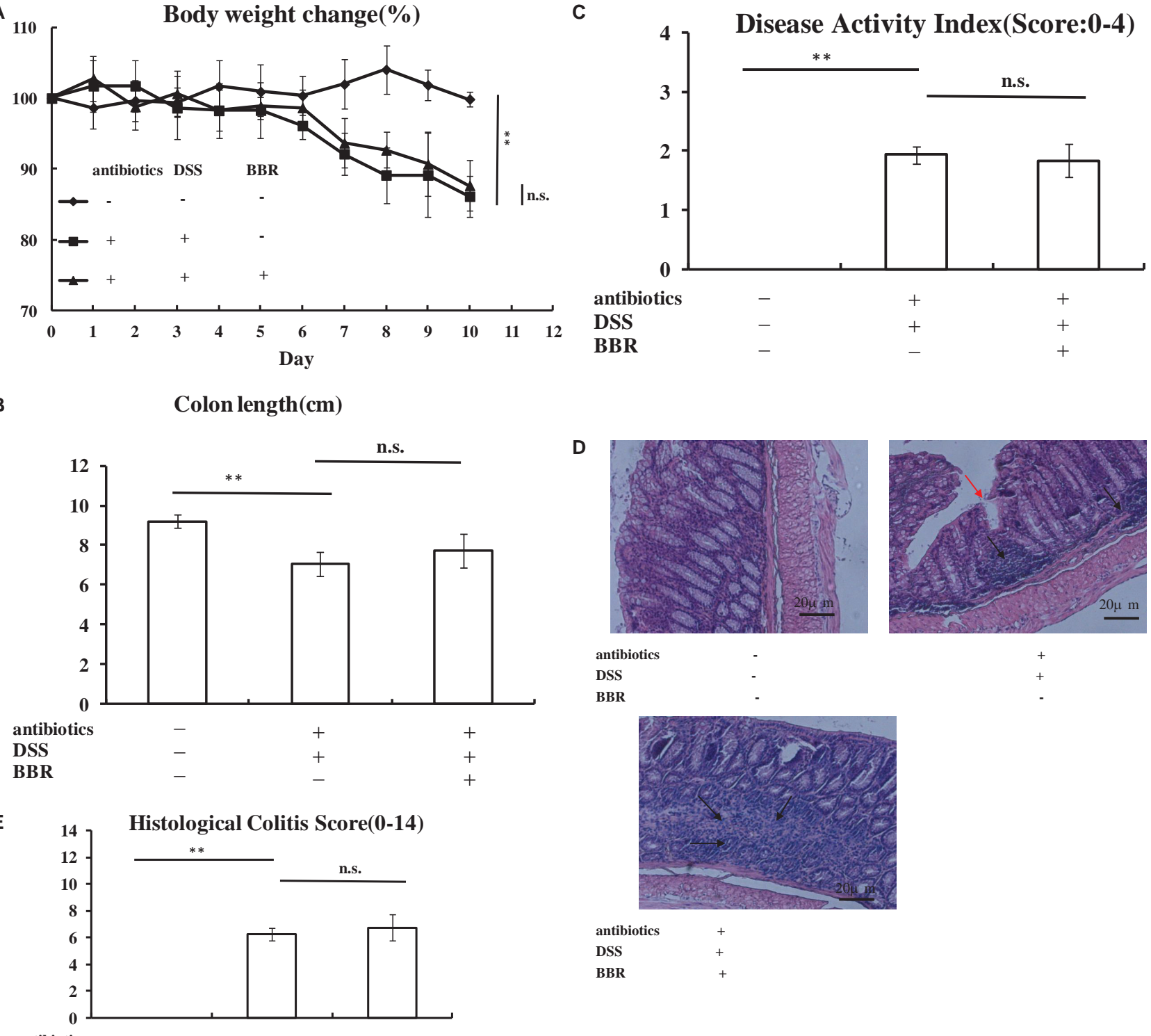

antibiotics

DSS

BBR

G Relative mRNA expression (fold to antibiotics+DSS+BBR-group)

$\mathbf{F}$

cytokines levels in colon tissue homogenate $(\mathrm{pg} / \mathrm{ml})$
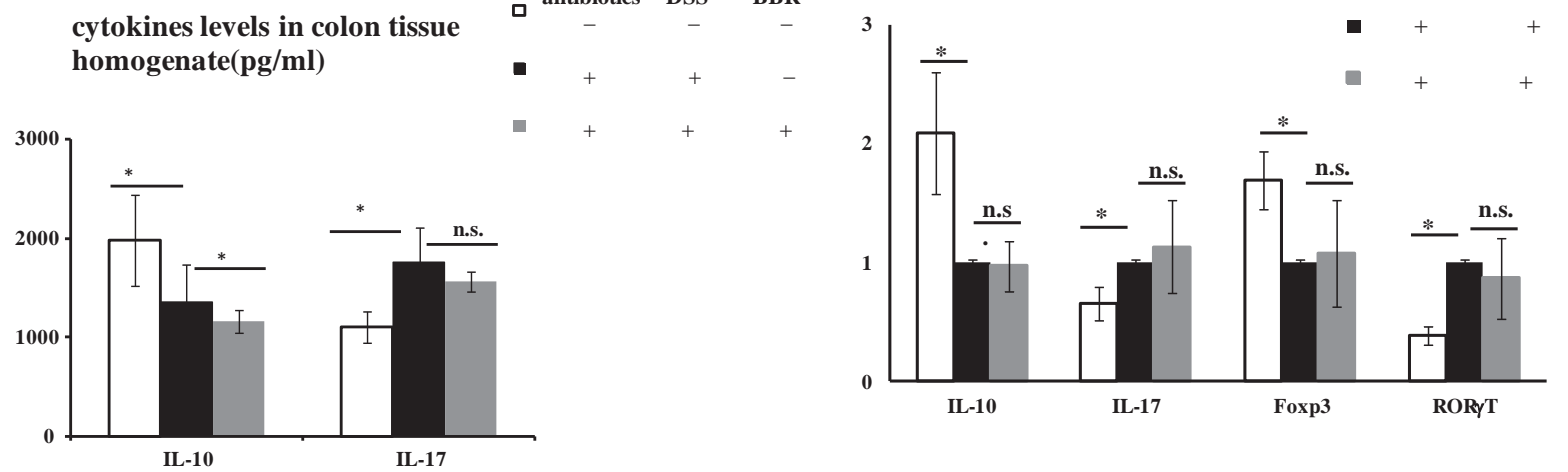

FIGURE 5 | Continued 
H a

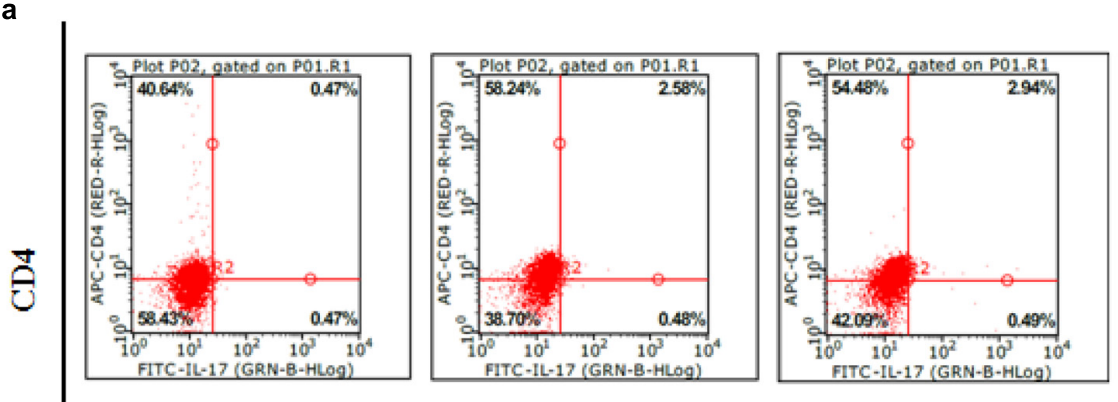

IL-17A

$\begin{array}{ll}\text { antibiotics } & - \\ \text { DSS } & - \\ \text { BBR } & -\end{array}$

b

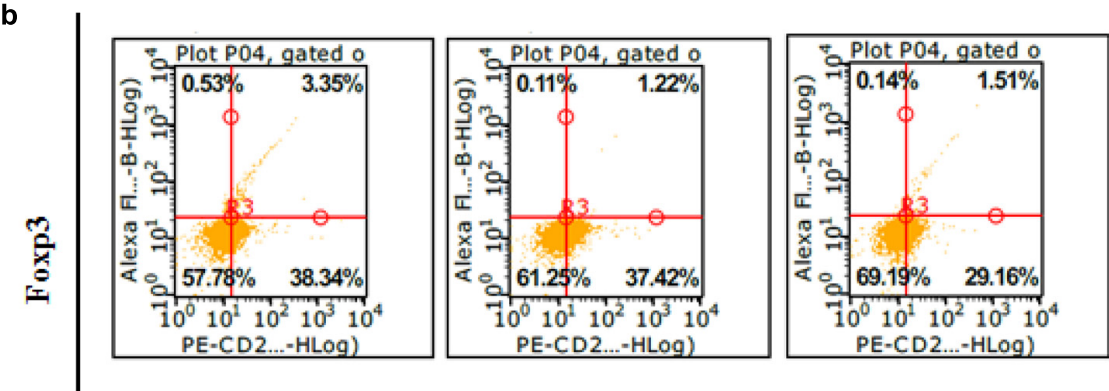

\section{CD25}

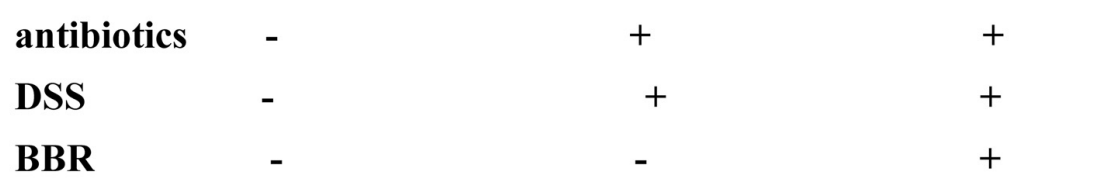

c

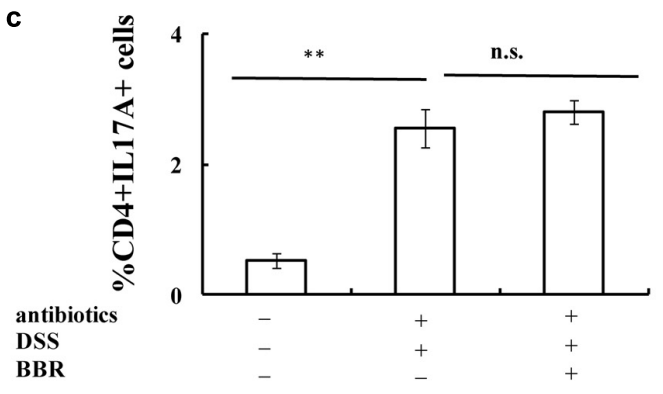

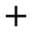

$+$

$+$

$+$

d

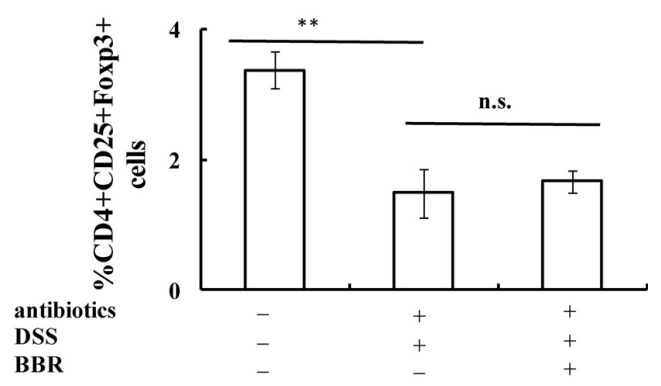

FIGURE $\mathbf{5}$ | After the depletion of gut microbiota, BBR could not improve Treg/Th17 balance in DSS-induced UC model mice. (A-C) After the depletion of gut microbiota, BBR could not affect body weight, shorten of colon length and DAl score in DSS-induced UC model mice; (D,E) H\&E staining (Arrows in red indicate mucosal necrosis, arrows in black indicate inflammatory cell infiltration; 20x) and histological colitis score showed that BBR could not reduce mucosal necrosis and inflammatory cell infiltration in colon after the depletion of gut microbiota; (F) After the depletion of gut microbiota, BBR treatment decreased IL-10 levels in colon tissue homogenate and BBR treatment could not affect IL-17 levels in colon tissue homogenate compared with antibiotics+DSS+BBR-group; (G) After the depletion of gut microbiota, BBR treatment could not affect the relative mRNA expression of $I L-10, I L-17$, Foxp3 and ROR $\gamma T$ in colonic mucosa compared with antibiotics+DSS+BBR- group (Fold to DSS+antibiotics+BBR- group); (H) After the depletion of gut microbiota, flow cytometric analysis showed that BBR treatment did not affect the numbers of $\mathrm{CD} 4^{+} \mathrm{IL} 17 \mathrm{~A}^{+}$(a: plot; c: graph) cells and CD4 ${ }^{+} \mathrm{CD} 25^{+} \mathrm{Foxp} 3^{+}$(b: plot; $\mathbf{d}$ : graph) in spleen compared with antibiotics+DSS+BBRgroup. Antibiotics-DSS-BBR-, antibiotics+DSS+BBR-, and antibiotics+DSS+ BBR+group ( $n=10$ per group, for A-G). Antibiotics-DSS-BBR-, antibiotics+DSS+BBR-, and antibiotics+DSS+ BBR+group $(n=6$ per group, $\mathbf{H})$. Data are presented as mean \pm SD. ${ }^{*} p<0.05,{ }^{* *} p<0.01$. 


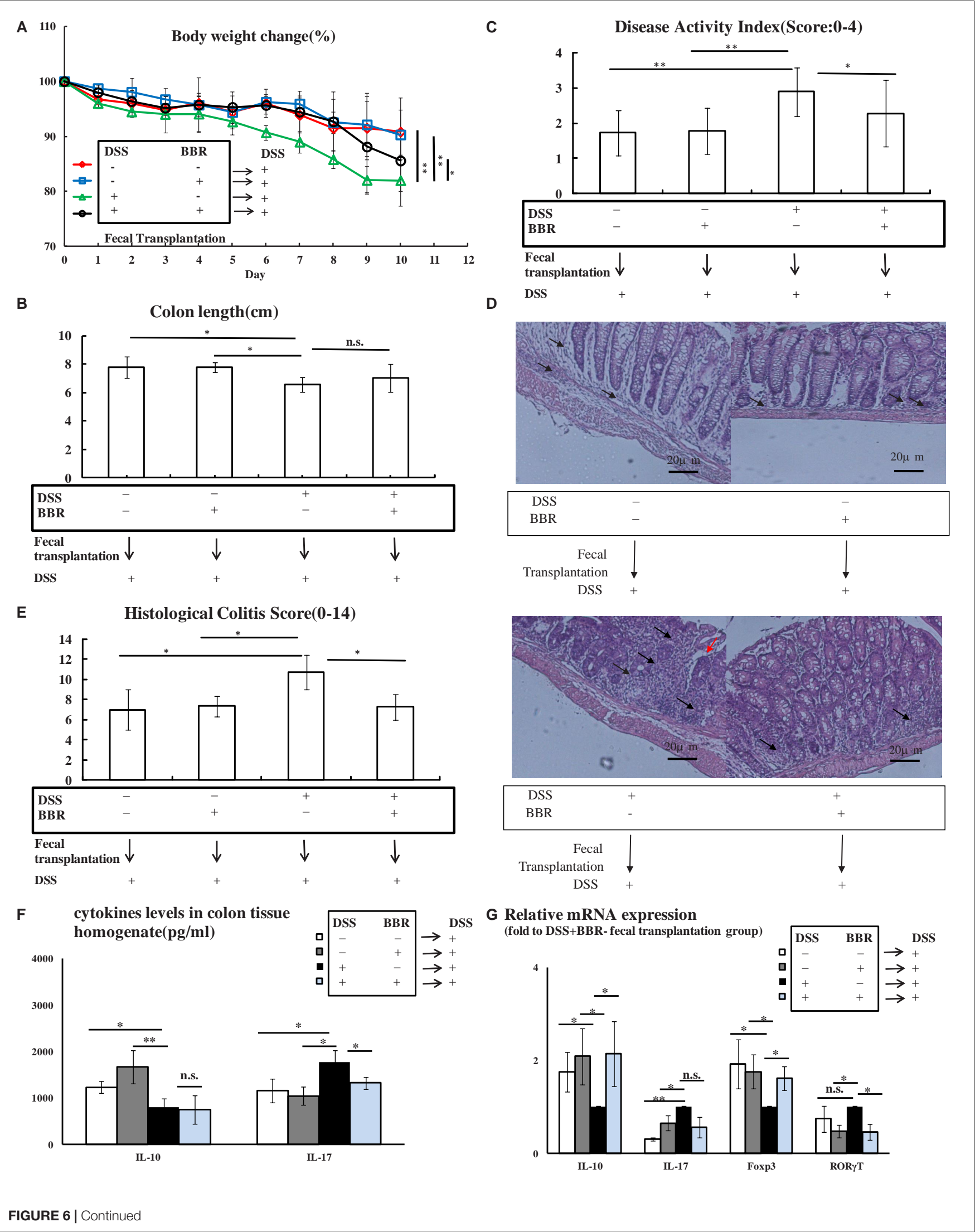


H a

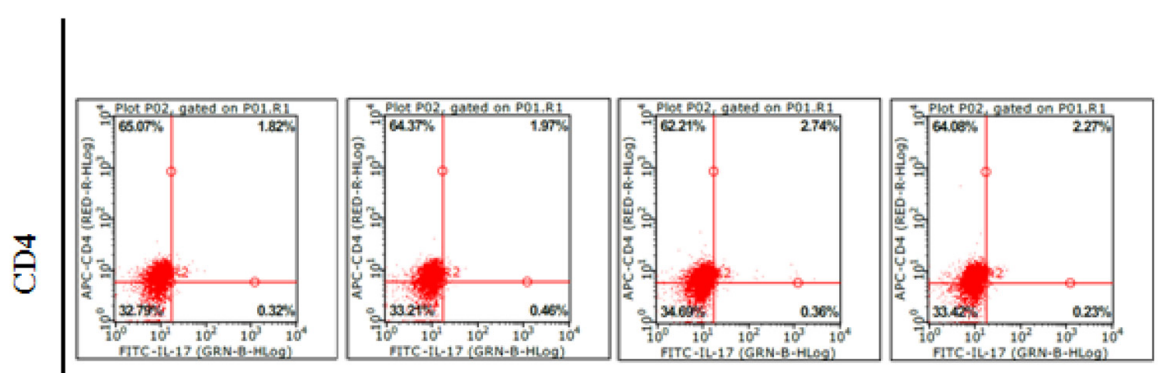

IL-17A

\begin{tabular}{|rrrrrr|}
\hline DSS & - & - & + & + \\
BBR & - & + & - & + \\
\hline Fecal & & $\mid$ & $\mid$ & $\downarrow$ \\
Transplantation & $\downarrow$ & $\downarrow$ & $\downarrow$ & + \\
DSS & + & + & + & & $\downarrow$
\end{tabular}

b

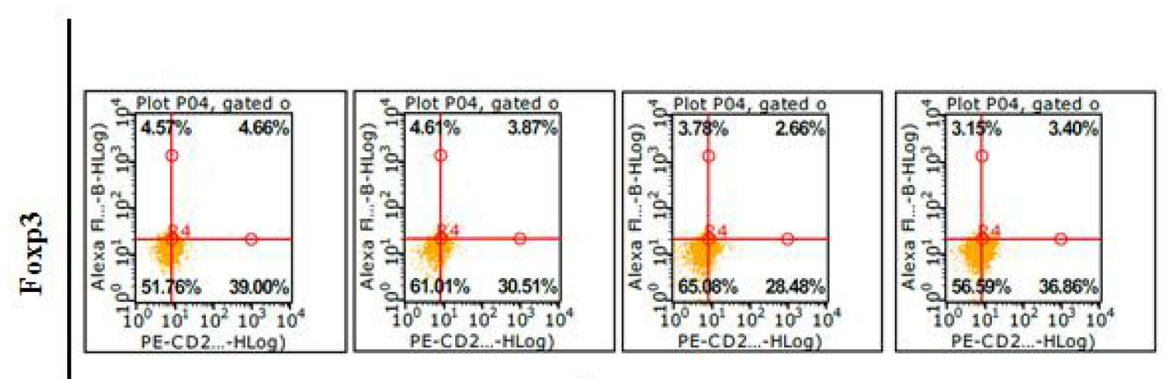

CD25

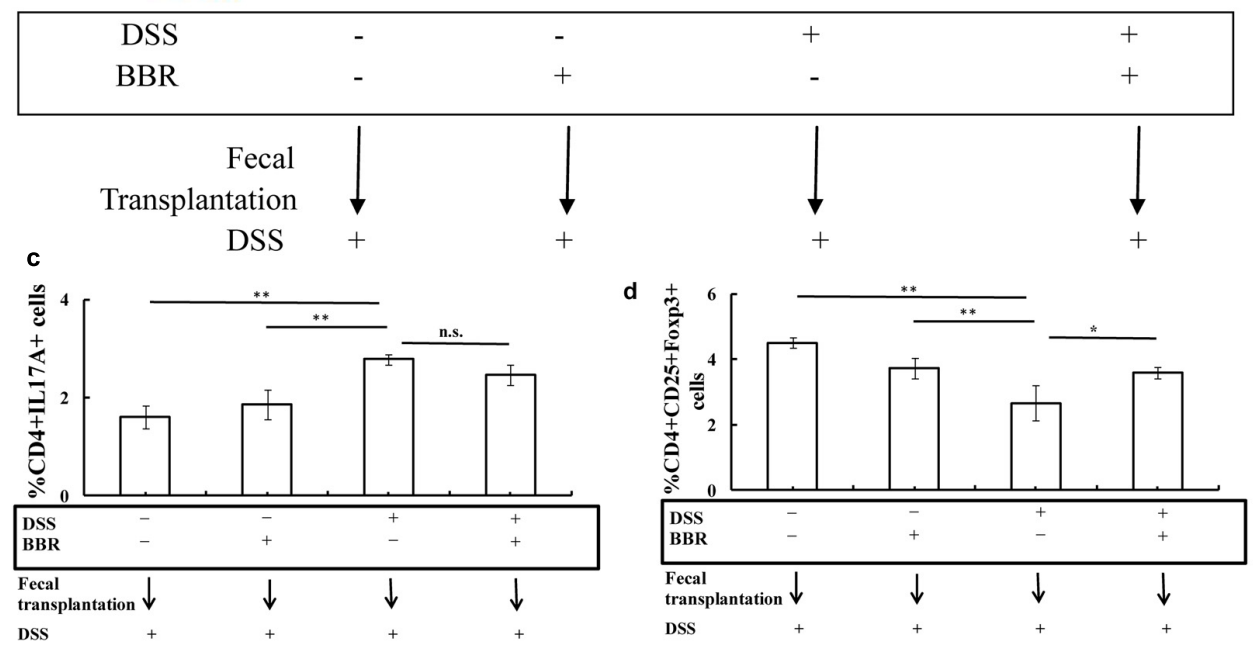

FIGURE 6 | Berberine fecal transplantation reduced UC by improving Treg/Th17 balance. (A,B) BBR fecal transplantation reduced body weight loss and decreased loss of colon length compared with DSS+BBR- fecal transplantation group; (C) Mice received BBR fecal transplantation had a lower DAl score compared with DSS+BBR- fecal transplantation group; (D,E) H\&E staining (Arrows in red indicate mucosal necrosis, arrows in black indicate inflammatory cell infiltration; 20x) and histological colitis score showed that BBR fecal transplantation reduced mucosal necrosis and inflammatory cell infiltration in colon; (F) BBR fecal transplantation increased IL-10 and decreased IL-17 levels of colon tissue homogenate compared with DSS+BBR- fecal transplantation group; (G) After BBR fecal transplantation, the relative mRNA expression of $I L-10$ and Foxp3 in colonic mucosa was upregulated whereas the relative mRNA expression of $I L-17$ and $R O R \gamma T$ in colonic mucosa was downregulated compared with DSS+BBR- fecal transplantation group (Fold to DSS+BBR- group); (H) Flow cytometric analysis showed that BBR fecal transplantation decreased the numbers of $\mathrm{CD}^{+}{ }^{+} \mathrm{L} 17 \mathrm{~A}^{+}$(a: plot; c: graph) cells and increased the numbers of $\mathrm{CD} 4^{+} \mathrm{CD}_{2} 5^{+}$Foxp3 ${ }^{+}$(b: plot; $\mathbf{d}$ : graph) in spleen compared with DSS+BBR- fecal transplantation group. DSS-BBR-, DSS-BBR+, DSS+BBR-, DSS+BBR+ fecal transplantation group $(n=7$ per group, for A-G). DSS-BBR-, DSS-BBR+, DSS+BBR-, DSS+BBR+ fecal transplantation group $(n=6$ per group, $\mathbf{H})$. Data are presented as mean \pm SD. ${ }^{*} p<0.05,{ }^{* *} p<0.01$. 
$(P<0.05, P<0.01$, respectively, Figure 6F). However, $I L$ 17 levels in colonic tissue homogenate was decreased in UC mice that received $\mathrm{DSS}-\mathrm{BBR}-, \mathrm{DSS}-\mathrm{BBR}+$, and $\mathrm{DSS}+\mathrm{BBR}+$ treated mice fecal transplantation as compared to $\mathrm{UC}$ mice that received $\mathrm{DSS}+\mathrm{BBR}-$ treated mice fecal transplantation $(P<0.05$, respectively, Figure 6F). $I L-10$ and Foxp3 gene expression in colonic mucosa were up-regulated in UC mice that received fecal transplants from DSS-BBR- DSS-BBR+, and $\mathrm{DSS}+\mathrm{BBR}+$ treated mice as compared to those that received fecal matter from DSS $+\mathrm{BBR}-$ treated mice $(P<0.05$, respectively, Figure 6G). $I L-17$ expression was down-regulated in UC mice given fecal matter from DSS-BBR- and DSS-BBR+ treated mice compared to UC mice administered feces from DSS+BBR - treated mice $(P<0.01, P<0.05$, respectively, Figure 6G). Moreover, $R O R \gamma T$ gene expression in colonic mucosa was down-regulated in UC mice given feces from DSS$\mathrm{BBR}+$ and $\mathrm{DSS}+\mathrm{BBR}+$ treated mice compared to those given feces from DSS+BBR - treated mice $(P<0.05$, Figure 6G). The number of $\mathrm{CD}^{+} \mathrm{IL}^{17 A^{+}}$(Th17) cells in spleen lymphocytes were decreased in UC mice receiving fecal transplantation from DSS-BBR - and DSS-BBR+ treated mice as compared to UC mice receiving fecal transplantation from DSS+BBRtreated mice $(P<0.01$, Figure $6 \mathbf{H})$, whereas the proportion of $\mathrm{CD}^{+} \mathrm{CD}^{+} 5^{+} \mathrm{Foxp}^{+}$(Treg) cells of spleen were decreased in $\mathrm{UC}$ mice receiving fecal transplantation from DSS-BBR-, $\mathrm{DSS}-\mathrm{BBR}+$, and $\mathrm{DSS}+\mathrm{BBR}+$ treated mice as compared to $\mathrm{UC}$ mice receiving fecal transplantation from DSS+BBRtreated mice $(P<0.01, P<0.01, P<0.05$, respectively, Figure 6H).

\section{DISCUSSION}

In the present study, the effect of BBR on DSS-induced UC was studied in vivo. Our results indicated a significant effect of BBR on UC, evidenced by a reduction in body weight loss, DAI score, mucosal necrosis, and infiltration of inflammatory cells as well as shortening of colon length. This work is in line with an earlier study (Shu et al., 2006).

Previous study has demonstrated that BBR could inhibit Th17 response in T-cell-mediated autoimmune diseases (Yang et al., 2013). It has also been reported that BBR could inhibit Th1/Th17 differentiation in UC model mice (Li et al., 2015). We further investigated the effect of BBR on Treg/Th17 balance, one of the important mechanisms in UC progression. Th17 cells differentiate from naïve $\mathrm{CD}^{+} \mathrm{T}$ cells through stimulation by transforming growth factor (TGF)- $\beta$ and $I L-6$. This process is mediated under the transcriptional regulation of retinoic acidrelated orphan receptor $(R O R \gamma T)$ (Ivanov et al., 2007; Ziegler and Buckner, 2009). Th17 cells produce pro-inflammatory cytokines including $I L-17, I L-21, I L-22, I L-23$, and $I L-25$, which contribute to the progression of UC (Korn et al., 2009; Weaver et al., 2013). Tregs could be distinguished into natural Tregs (nTregs) and inducible Tregs (iTregs) due to their different origins. nTregs are derived from the thymus with the function of suppressing the innate immune response (van Wijk and Cheroutre, 2010), whereas iTregs differentiate from naïve $\mathrm{CD}^{+} \mathrm{T}$ cells in the periphery when stimulated by specific antigens and they are crucial in the suppressive control of adaptive immunity (van Wijk and Cheroutre, 2010). The differentiation of both iTregs and nTregs are regulated by the transcription factor forkhead box P3 (Foxp3). Mature Tregs express high levels of Foxp3 (Fontenot et al., 2003; Bacchetta et al., 2006). Moreover, both iTregs and nTregs could secrete inhibitory cytokines including $I L-10, I L-$ 35 , and TGF- $\beta$, which could suppress both adaptive and innate immune responses (Jonuleit and Schmitt, 2003). Studies have shown that increased Th17 cells produced high levels of $I L$ 17, promoting inflammation. However, differentiation of Tregs was inhibited, triggering the reduction of immunosuppressive cytokines including TGF- $\beta$ and $I L-10$ in the mucosa (Fujino et al., 2003; Sugihara et al., 2010; Schiering et al., 2014). Furthermore, improving Treg/Th17 balance contributed to the re-establishment of intestinal immune homeostasis and reduced UC (Bai et al., 2009; Lee et al., 2015). In this study, BBR treatment improved the Treg/Th17 balance in DSS-induced UC model, consistent with previous studies.

It has been demonstrated that Bacteroides could interact with Treg to promote $I L-10$ production by producing metabolites (Smith et al., 2013; Luo et al., 2017). Colonization of segmented filamentous bacteria could induce Th17 cell differentiation in the intestinal lamina propria (Ivanov et al., 2009). We also investigated changes in microbiological composition using highthroughput sequencing. Our results showed that DSS treatment could reduce the alpha diversity of the gut microbiota community based on a lower Shannon index compared with DSS-BBRtreated mice. Moreover, PCoA analysis and the system clustering tree showed significant distances between each group, consistent with the previous study that DSS treatment could change the beta diversity of the gut microbiota community (Yang et al., 2017). Interestingly, the distances in PCoA analysis and the system clustering tree were close between the DSS+BBR+ and DSS-BBR- groups. Our results also showed that BBR could decrease the relative abundance of Desulfovibrio and increase Eubacterium strains after DSS treatment. In agreement with a previous study, Desulfovibrio bacterial species were increased in UC and Desulfovibrio were harmful to colonic epithelial cells (Rowan et al., 2010). Eubacterium limosum could ameliorate experimental colonic inflammation by producing butyrate to increase mucosal integrity (Kanauchi et al., 2006). Additionally, our results also revealed a significant increase of Bacteroides after BBR treatment.

We also investigated whether gut microbiota are involved in the regulation of the Treg/Th17 balance by BBR in UC. The depletion of gut microbiota through antibiotics treatment is commonly used in order to understand the role of gut microbiota (Elinav et al., 2011; Hoban et al., 2017). In our study, analysis of fecal total DNA showed that gut microbiota were depleted after treatment with a combination of antibiotics. Due to the hepatotoxicity and nephrotoxicity of antibiotics, we also measured the long-term effect of antibiotics on liver and kidney function in mice. The use of antibiotics did not affect serum ALT, AST, Cr, or BUN levels, nor did it affect the histological profiles of the liver, kidney, small intestine, or colon. It was also observed that BBR had a decreased effect on UC following depletion of 
gut microbiota, indicating that BBR treatment does not influence Treg/Th17 balance after depletion of gut microbiota.

Fecal transplantation is a novel method for studying the relationship between herbs and microbiota. Research has shown that mice administered Ganoderma lucidum fecal transplants had reduced high fat diet-induced obesity, indicating that gut microbiota is a key factor for G. lucidum in reducing obesity (Chang et al., 2017). In our study, fecal transplantation was carried out in order to assess the role played by gut microbiota during treatment of UC with BBR. Our findings indicated that fecal samples from BBR treated mice resulted in reduced UC and modulated Treg/Th17 balance.

In the present study, we first determined Treg and Th17 activities in UC model mice treated with BBR. Our results indicated that BBR influenced the Treg/Th17 balance in UC model mice. Secondly, we found that the effect of BBR on the Treg/Th17 balance in UC model mice was decreased after gut microbiota were depleted through antibiotics treatment. Finally, when fecal samples from mice that received BBR treatment were

\section{REFERENCES}

Anderson, J. L., Edney, R. J., and Whelan, K. (2012). Systematic review: faecal microbiota transplantation in the management of inflammatory bowel disease. Aliment. Pharmacol. Ther. 36, 503-516. doi: 10.1111/j.1365-2036.2012.05220.x

Bacchetta, R., Passerini, L., Gambineri, E., Dai, M., Allan, S. E., Perroni, L., et al. (2006). Defective regulatory and effector $\mathrm{T}$ cell functions in patients with FOXP3 mutations. J. Clin. Invest. 116, 1713-1722. doi: 10.1172/jci25112

Bai, A., Lu, N., Guo, Y., Liu, Z., Chen, J., and Peng, Z. (2009). All-trans retinoic acid down-regulates inflammatory responses by shifting the Treg/Th17 profile in human ulcerative and murine colitis. J. Leukoc. Biol. 86, 959-969. doi: 10.1016/s0016-5085(09)61870-9

Borody, T. J., Paramsothy, S., and Agrawal, G. (2013). Fecal microbiota transplantation: indications, methods, evidence, and future directions. Curr. Gastroenterol. Rep. 15:337. doi: 10.1007/s11894-013-0337-1

Borody, T. J., Warren, E. F., Leis, S., Surace, R., and Ashman, O. (2003). Treatment of ulcerative colitis using fecal bacteriotherapy. J. Clin. Gastroenterol. 37, 42-47. doi: 10.1097/00004836-200307000-00012

Chang, C. J., Lin, C. S., Lu, C. C., Martel, J., Ko, Y. F., Ojcius, D. M., et al. (2017). Corrigendum: Ganoderma lucidum reduces obesity in mice by modulating the composition of the gut microbiota. Nat. Commun. 8:16130. doi: 10.1038/ ncomms 16130

Chen, C., Yu, Z., Li, Y., Fichna, J., and Storr, M. (2014). Effects of berberine in the gastrointestinal tract - a review of actions and therapeutic implications. Am. J. Chin. Med. 42, 1053-1070. doi: 10.1142/s0192415x14500669

Citri, A., Pang, Z. P., Sudhof, T. C., Wernig, M., and Malenka, R. C. (2011). Comprehensive qPCR profiling of gene expression in single neuronal cells. Nat. Protoc. 7, 118-127. doi: 10.1038/nprot.2011.430

Cui, H. H., Chen, C. L., Wang, J. D., Yang, Y. J., Cun, Y., Wu, J. B., et al. (2004). Effects of probiotic on intestinal mucosa of patients with ulcerative colitis. World J. Gastroenterol. 10, 1521-1525. doi: 10.3748/wjg.v10.i10.1521

Di Paolo, M. C., Paoluzi, O. A., Pica, R., Iacopini, F., Crispino, P., Rivera, M., et al. (2001). Sulphasalazine and 5-aminosalicylic acid in long-term treatment of ulcerative colitis: report on tolerance and side-effects. Dig. Liver Dis. 33, 563-569. doi: 10.1016/s1590-8658(01)80108-0

Elinav, E., Strowig, T., Kau, A. L., Henao-Mejia, J., Thaiss, C. A., Booth, C. J., et al. (2011). NLRP6 inflammasome regulates colonic microbial ecology and risk for colitis. Cell 145, 745-757. doi: 10.1016/j.cell.2011.04.022

Feng, L., Shao-Min, L. I., Dai, L. J., Chun-Mei, L. I., Yan, L. I., Fei, W. U., et al. (2015). Effects of polysaccharides from Portulaca oleracea L. on intestinal mucosal cytokines and intestinal flora in mice with ulcerative colitis. Chin. J. Microecol. 39, 88-91. transplanted into UC model mice, we found that the imbalance of Treg/Th17 in recipient mice was improved. In conclusion, gut microbiota plays an important role during UC treatment as it affects the efficacy of BBR. The mechanism for this effect could be related to regulation of the Treg/Th17 balance.

\section{AUTHOR CONTRIBUTIONS}

HC wrote the manuscript. YC, XC, JiL, LW, HC, and SZ conducted animal experiments. BJ, JuL, YC, and XZ finished molecular bioassays. YB and $\mathrm{PZ}$ provided technical guidance for the whole work. All the authors approved the final draft.

\section{FUNDING}

This work was supported by National Natural Science Foundation of China (Nos. 81573707 and 81673707).

Fontenot, J. D., Gavin, M. A., and Rudensky, A. Y. (2003). Foxp3 programs the development and function of $\mathrm{CD} 4^{+} \mathrm{CD} 25^{+}$regulatory T cells. Nat. Immunol. 4 , 330-336. doi: 10.1038/ni904

Fujino, S., Andoh, A., Bamba, S., Ogawa, A., Hata, K., Araki, Y., et al. (2003). Increased expression of interleukin 17 in inflammatory bowel disease. Gut 52, 65-70. doi: 10.1136/gut.52.1.65

Harrington, L. E., Hatton, R. D., Mangan, P. R., Turner, H., Murphy, T. L., Murphy, K. M., et al. (2005). Interleukin 17-producing $\mathrm{CD}^{+}{ }^{+}$effector $\mathrm{T}$ cells develop via a lineage distinct from the $\mathrm{T}$ helper type 1 and 2 lineages. Nat. Immunol. 6, 1123-1132. doi: 10.1038/ni1254

Hoban, A. E., Stilling, R. M., Moloney, G. M., Moloney, R. D., Shanahan, F., Dinan, T. G., et al. (2017). Microbial regulation of microRNA expression in the amygdala and prefrontal cortex. Microbiome 5:102. doi: 10.1186/s40168-0170321-3

Hoque, S. S., Poxton, I. R., and Ghosh, S. (2000). Gut bacteria and ulcerative colitis - A broken tolerance. Gastroenterology 118:A807. doi: 10.1016/s00165085(00)85369-x

Ivanov, I. I., Atarashi, K., Manel, N., Brodie, E. L., Shima, T., Karaoz, U., et al. (2009). Induction of intestinal Th17 cells by segmented filamentous bacteria. Cell 139, 485-498. doi: 10.1016/j.cell.2009.09.033

Ivanov, I. I., Zhou, L., and Littman, D. R. (2007). Transcriptional regulation of Th17 cell differentiation. Semin. Immunol. 19, 409-417. doi: 10.1016/j.smim.2007. 10.011

Jonuleit, H., and Schmitt, E. (2003). The regulatory T cell family: distinct subsets and their interrelations. J. Immunol. 171, 6323-6327. doi: 10.4049/jimmunol. 171.12.6323

Kanauchi, O., Fukuda, M., Matsumoto, Y., Ishii, S., Ozawa, T., Shimizu, M., et al. (2006). Eubacterium limosum ameliorates experimental colitis and metabolite of microbe attenuates colonic inflammatory action with increase of mucosal integrity. World J. Gastroenterol. 12, 1071-1077. doi: 10.3748/wjg.v12.i7. 1071

Kihara, N., de la Fuente, S. G., Fujino, K., Takahashi, T., Pappas, T. N., and Mantyh, C. R. (2003). Vanilloid receptor-1 containing primary sensory neurones mediate dextran sulphate sodium induced colitis in rats. Gut 52, 713-719. doi: 10.1136/ gut.52.5.713

Korn, T., Bettelli, E., Oukka, M., and Kuchroo, V. K. (2009). IL-17 and Th17 Cells. Annu. Rev. Immunol. 27, 485-517. doi: 10.1146/annurev.immunol.021908. 132710

Kunde, S., Pham, A., Bonczyk, S., Crumb, T., Duba, M., Conrad, H., et al. (2013). Safety, tolerability, and clinical response after fecal transplantation in children and young adults with ulcerative colitis. J. Pediatr. Gastroenterol. Nutr. 56, 597-601. doi: 10.1097/mpg.0b013e318292fa0d 
Lee, S. Y., Lee, S. H., Yang, E. J., Kim, E. K., Kim, J. K., Shin, D. Y., et al. (2015). Metformin ameliorates inflammatory bowel disease by suppression of the STAT3 signaling pathway and regulation of the between Th17/Treg balance. PLoS One 10:e0135858. doi: 10.1371/journal.pone.013 5858

Li, C., Xi, Y., Li, S., Zhao, Q., Cheng, W., Wang, Z., et al. (2015). Berberine ameliorates TNBS induced colitis by inhibiting inflammatory responses and Th1/Th17 differentiation. Mol. Immunol. 67, 444-454. doi: 10.1016/j.molimm. 2015.07.013

Li, F., Hao, X., Chen, Y., Bai, L., Gao, X., Lian, Z., et al. (2017). The microbiota maintain homeostasis of liver-resident $\gamma \delta \mathrm{T}-17$ cells in a lipid antigen/CD1ddependent manner. Nat. Commun. 7:13839. doi: 10.1038/ncomms 15265

Livak, K. J., and Schmittgen, T. D. (2001). Analysis of relative gene expression data using real-time quantitative PCR and the $2^{-\Delta \Delta C_{\mathrm{T}}}$ method. Methods 25, 402-408. doi: 10.1006/meth.2001.1262

Locatelli, M., Ferrante, C., Carradori, S., Secci, D., Leporini, L., Chiavaroli, A., et al. (2017). Optimization of aqueous extraction and biological activity of Harpagophytum procumbens root on ex vivo rat colon inflammatory model. Phytother. Res. 31, 937-944. doi: 10.1002/ptr.5821

Luo, A., Leach, S. T., Barres, R., Hesson, L. B., Grimm, M. C., and Simar, D. (2017). The microbiota and epigenetic regulation of T helper 17/regulatory T cells: in search of a balanced immune system. Front. Immunol. 8:417. doi: 10.3389/fimmu.2017.00417

Matsunaga, T., Hashimoto, S., Yamamoto, N., Kawasato, R., Shirasawa, T., Goto, A., et al. (2017). Protective effect of daikenchuto on dextran sulfate sodiuminduced colitis in mice. Gastroenterol. Res. Pract. 2017:1298263. doi: 10.1155/ 2017/1298263

Menghini, L., Ferrante, C., Leporini, L., Recinella, L., Chiavaroli, A., Leone, S., et al. (2016). An hydroalcoholic chamomile extract modulates inflammatory and immune response in HT29 cells and isolated rat colon. Phytother. Res. 30, 1513-1518. doi: 10.1002/ptr.5655

Moayyedi, P., Surette, M. G., Kim, P. T., Libertucci, J., Wolfe, M., Onischi, C., et al. (2015). Fecal microbiota transplantation induces remission in patients with active ulcerative colitis in a randomized controlled trial. Gastroenterology 149, 102.e6-109.e6. doi: 10.1053/j.gastro

Rijnierse, A., Koster, A. S., Nijkamp, F. P., and Kraneveld, A. D. (2006). TNF-alpha is crucial for the development of mast cell-dependent colitis in mice. Am. J. Physiol. Gastrointest. Liver Physiol. 291, G969-G976. doi: 10.1152/ajpgi.00146. 2006

Rowan, F., Docherty, N. G., Murphy, M., Murphy, B., Calvin Coffey, J., and O'Connell, P. R. (2010). Desulfovibrio bacterial species are increased in ulcerative colitis. Dis. Colon Rectum 53, 1530-1536. doi: 10.1007/DCR. 0b013e3181fle620

Schiering, C., Krausgruber, T., Chomka, A., Frohlich, A., Adelmann, K., Wohlfert, E. A., et al. (2014). The alarmin IL-33 promotes regulatory T-cell function in the intestine. Nature 513, 564-568. doi: 10.1038/nature13577

Seidelin, J. B., Bjerrum, J. T., Coskun, M., Widjaya, B., Vainer, B., and Nielsen, O. H. (2010). IL-33 is upregulated in colonocytes of ulcerative colitis. Immunol. Lett. 128, 80-85. doi: 10.1016/j.imlet

Shen, J., Zuo, Z. X., and Mao, A. P. (2014). Effect of probiotics on inducing remission and maintaining therapy in ulcerative colitis, Crohn's disease, and pouchitis: meta-analysis of randomized controlled trials. Inflamm. Bowel Dis. 20, 21-35. doi: 10.1097/01.MIB.0000437495.30 052.be
Shu, D. Z., Wan, X. H., Liu, H. R., Yang, J. Q., and Zhou, Q. X. (2006). Effect of berberine chloride on experimental murine colitis induced by dextran sulfate sodium. J. Chin. Pharm. Sci. 15, 182-187.

Smith, P. M., Howitt, M. R., Panikov, N., Michaud, M., Gallini, C. A., Bohlooly, Y. M., et al. (2013). The microbial metabolites, short-chain fatty acids, regulate colonic Treg cell homeostasis. Science 341, 569-573. doi: 10.1126/science. 1241165

Sugihara, T., Kobori, A., Imaeda, H., Tsujikawa, T., Amagase, K., Takeuchi, K., et al. (2010). The increased mucosal mRNA expressions of complement C3 and interleukin-17 in inflammatory bowel disease. Clin. Exp. Immunol. 160, 386-393. doi: 10.1111/j.1365-2249.2010.04093.x

van Wijk, F., and Cheroutre, H. (2010). Mucosal T cells in gut homeostasis and inflammation. Expert Rev. Clin. Immunol. 6, 559-566. doi: 10.1586/eci.10.34

Wang, X., Sun, Y., Zhao, Y., Ding, Y., Zhang, X., Kong, L., et al. (2016). Oroxyloside prevents dextran sulfate sodium-induced experimental colitis in mice by inhibiting NF-кB pathway through PPAR $\gamma$ activation. Biochem. Pharmacol. 106, 70-81. doi: 10.1016/j.bcp.2016.02.019

Weaver, C. T., Elson, C. O., Fouser, L. A., and Kolls, J. K. (2013). The Th17 pathway and inflammatory diseases of the intestines, lungs, and skin. Annu. Rev. Pathol. 8, 477-512. doi: 10.1016/j.bcp.2016.02.019

Yang, Y., Chen, G., Yang, Q., Ye, J., Cai, X., Tsering, P., et al. (2017). Gut microbiota drives the attenuation of dextran sulphate sodium-induced colitis by Huangqin decoction. Oncotarget 8, 48863-48874. doi: 10.18632/oncotarget.16458

Yang, Y., Qi, J., Wang, Q., Du, L., Zhou, Y., Yu, H., et al. (2013). Berberine suppresses Th17 and dendritic cell responses. Invest. Ophthalmol. Vis. Sci. 54, 2516-2522. doi: 10.18632/oncotarget.16458

Yao, H., Zheng, P., Xishi, L. I., Jiansheng, W. U., and Guoyou, W. U. (2013). Rectification of disorder of serum IL-33 and IL-10 and intestinal flora of patients with ulcerative colitis by Yiqi Yukui decotion. Chin. Arch. Tradit. Chin. Med. 6, $1449-1451$.

Zhang, X., Zhao, Y., Zhang, M., Pang, X., Xu, J., Kang, C., et al. (2012). Structural changes of gut microbiota during berberine-mediated prevention of obesity and insulin resistance in high-fat diet-fed rats. PLoS One 7:e42529. doi: 10.1371/ journal.pone.0042529

Zhao, H. M., Huang, X. Y., Zuo, Z. Q., Pan, Q. H., Ao, M. Y., Zhou, F., et al. (2013). Probiotics increase $T$ regulatory cells and reduce severity of experimental colitis in mice. World J. Gastroenterol. 19, 742-749. doi: 10.3748/wjg.v19.i5.742

Zhao, Q., Wang, S., and Xia, L. (2013). Gancao xiexin decoction in the treatment of relapsing ulcerative colitis:clinical observation and the effects of intestinal microflora and serum interleukin 6,10. Chin. Arch. Tradit. Chin. Med. 4, 944-946.

Ziegler, S. F., and Buckner, J. H. (2009). FOXP3 and the regulation of Treg/Th17 differentiation. Microbes Infect. 11, 594-598. doi: 10.1016/j.micinf.2009.04.002

Conflict of Interest Statement: The authors declare that the research was conducted in the absence of any commercial or financial relationships that could be construed as a potential conflict of interest.

Copyright () 2018 Cui, Cai, Wang, Jia, Li, Zhao, Chu, Lin, Zhang, Bian and Zhuang. This is an open-access article distributed under the terms of the Creative Commons Attribution License (CC BY). The use, distribution or reproduction in other forums is permitted, provided the original author(s) and the copyright owner are credited and that the original publication in this journal is cited, in accordance with accepted academic practice. No use, distribution or reproduction is permitted which does not comply with these terms. 